\title{
Multitriangulations, Pseudotriangulations and Primitive Sorting Networks
}

\author{
Vincent Pilaud • Michel Pocchiola
}

Received: 27 September 2010 / Revised: 3 February 2012 / Accepted: 5 February 2012 /

Published online: 1 March 2012

(C) Springer Science+Business Media, LLC 2012

\begin{abstract}
We study the set of all pseudoline arrangements with contact points which cover a given support. We define a natural notion of flip between these arrangements and study the graph of these flips. In particular, we provide an enumeration algorithm for arrangements with a given support, based on the properties of certain greedy pseudoline arrangements and on their connection with sorting networks. Both the running time per arrangement and the working space of our algorithm are polynomial.

As the motivation for this work, we provide in this paper a new interpretation of both pseudotriangulations and multitriangulations in terms of pseudoline arrangements on specific supports. This interpretation explains their common properties and leads to a natural definition of multipseudotriangulations, which generalizes both. We study elementary properties of multipseudotriangulations and compare them to iterations of pseudotriangulations.
\end{abstract}

Keywords Pseudoline arrangement · Pseudotriangulation · Multitriangulation · Flip · Sorting network · Enumeration algorithm

An extended abstract of this paper was presented in the 25th European Workshop on Computational Geometry (Brussels, March 2009) [27]. The main results of this paper also appeared in Chap. 3 of the first author's Ph.D. dissertation [26].

V. Pilaud (凶)

CNRS \& LIX (UMR 7161), École Polytechnique, Palaiseau, France

e-mail: vincent.pilaud@lix.polytechnique.fr

url: http://www.lix.polytechnique.fr/ pilaud/

M. Pocchiola

Institut de Mathématiques de Jussieu (UMR 7586), Université Pierre et Marie Curie, Paris, France

e-mail: pocchiola@math.jussieu.fr

url: http://people.math.jussieu.fr/ pocchiola/ 


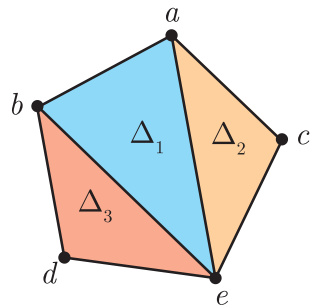

(a)

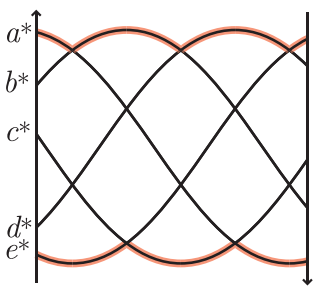

(b)

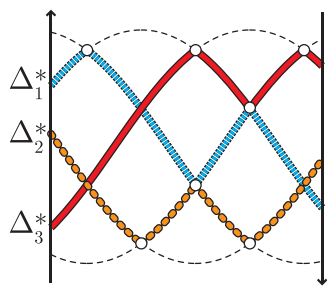

(c)

Fig. 1 (a) A triangulation $T$ of a convex point set $P$. (b) The dual pseudoline arrangement $P^{*}$ of $P$ (whose first level is highlighted). (c) The dual pseudoline arrangement $T^{*}$ of $T$

\section{Introduction}

The original motivation for this paper is the interpretation of certain families of planar geometric graphs in terms of pseudoline arrangements. As an introductory illustration, we present this interpretation on the family of triangulations of a convex polygon.

Let $P$ be a finite point set in convex position in the Euclidean plane $\mathbb{R}^{2}$. A triangulation $T$ of $P$ is a maximal crossing-free set of edges on $P$, or equivalently, a decomposition of the convex hull of $P$ into triangles with vertices in $P$. See Fig. 1(a). A (strict) bisector of a triangle $\Delta$ of $T$ is a line which passes through a vertex of $\Delta$ and (strictly) separates its other two vertices. Observe that any triangle has a unique bisector parallel to any direction. Moreover any two triangles of $T$ have a unique common strict bisector, and possibly an additional non-strict bisector if they share an edge.

We now switch to the line space $\mathcal{M}$ of the Euclidean plane $\mathbb{R}^{2}$. Remember that $\mathcal{M}$ is a Möbius strip homeomorphic to the quotient space $\mathbb{R}^{2} /(\theta, d) \sim(\theta+\pi,-d)$ via the parametrization of an oriented line by its angle $\theta$ with the horizontal axis and its algebraic distance $d$ to the origin (see Sect. 3 for details). For pictures, we represent $\mathcal{M}$ as a vertical band whose boundaries are identified in opposite directions. The dual of a point $p \in P$ is the set of all lines of $\mathbb{R}^{2}$ passing through $p$. It is a pseudoline of $\mathcal{M}$, i.e. a non-separating simple closed curve. The dual of $P$ is the set $P^{*}:=\left\{p^{*} \mid p \in P\right\}$. It is a pseudoline arrangement of $\mathcal{M}$, since any two pseudolines $p^{*}, q^{*}$ of $P^{*}$ cross precisely once at the line $(p q)$. Call first level of $P^{*}$ the boundary of the external face of the complement of $P^{*}$. It corresponds to the supporting lines of the convex hull of $P$. See Fig. 1(b).

As illustrated on Fig. 1(c), we observe after $[32,33]$ that:

(i) the set $\Delta^{*}$ of all bisectors of a triangle $\Delta$ of $T$ is a pseudoline of $\mathcal{M}$;

(ii) the dual pseudolines $\Delta_{1}^{*}, \Delta_{2}^{*}$ of any two triangles $\Delta_{1}, \Delta_{2}$ of $T$ have a unique crossing point (the unique common strict bisector of $\Delta_{1}$ and $\Delta_{2}$ ) and possibly a contact point (when $\Delta_{1}$ and $\Delta_{2}$ share a common edge);

(iii) the set $T^{*}:=\left\{\Delta^{*} \mid \Delta\right.$ triangle of $\left.T\right\}$ is a pseudoline arrangement with contact points; and

(iv) $T^{*}$ covers $P^{*}$ minus its first level. 
We furthermore prove in this paper that this interpretation is bijective: any pseudoline arrangement with contact points supported by $P^{*}$ minus its first level is the dual pseudoline arrangement $T^{*}$ of a triangulation $T$ of $P$.

Motivated by this interpretation, we study the set of all pseudoline arrangements with contact points which cover a given support in the Möbius strip. We define a natural notion of flip between them, and study the graph of these flips. In particular, we provide an enumeration algorithm for arrangements with a given support (similar to the enumeration algorithm of [7] for pseudotriangulations), based on certain greedy pseudoline arrangements and their connection with primitive sorting networks [23, Sect. 5.3.4, 10, 24]. The running time per arrangement and the working space of our algorithm are both polynomial.

We are especially interested in the following particular situation. Let $L$ be a pseudoline arrangement and $k$ be a positive integer. We call $k$-pseudotriangulation of $L$ any pseudoline arrangement with contact points that covers $L$ minus its first $k$ levels. These objects provide dual interpretations for two, until now unrelated, classical generalizations of triangulations of a convex polygon (see Sect. 3 for the definitions and basic properties of these geometric graphs):

Pseudotriangulations $(k=1)$. Introduced for the study of the visibility complex of a set of disjoint convex obstacles in the plane [34, 35], pseudotriangulations were used in different contexts such as motion planning and rigidity theory [15, 42]. Their combinatorial and geometric structure has been extensively studied in the last decade (number of pseudotriangulations [1, 2], polytope of pseudotriangulations [36], algorithmic issues [5, 7, 16], etc.). See [37] for a detailed survey on the subject. As far as pseudotriangulations are concerned, this paper has two main applications: it proves the dual characterization of pseudotriangulations in terms of pseudoline arrangements and provides an interpretation of greedy pseudotriangulations in terms of sorting networks, leading to a new proof of the greedy flip property for points [3, 7, 34]. The objects studied in this paper have a further (algorithmic) motivation: as a first step to compute the dual arrangement of a set of disjoint convex bodies described only by its chirotope, Habert and Pocchiola raise in [16] the question to compute efficiently a pseudotriangulation of a pseudotriangulation of the set, i.e. a 2-pseudotriangulation.

Multitriangulations (convex position). Introduced in the context of extremal theory for geometric graphs [9], multitriangulations were then studied for their combinatorial structure $[11,12,20,25]$. The study of stars in multitriangulations [28], generalizing triangles for triangulations, naturally leads to interpret multitriangulations as multipseudotriangulations of points in convex position. As far as we know, this paper provides the first interpretation of multitriangulations in terms of pseudoline arrangements on the Möbius strip.

The paper is organized as follows. Section 2 is devoted to the study of all pseudoline arrangements with contact points covering a given support. We define the flip and the greedy pseudoline arrangements (2.2) whose properties yield the enumeration algorithm for pseudoline arrangements with a given support (2.3).

In Sect. 3, we prove that the pseudotriangulations of a finite planar point set $P$ in general position correspond to the pseudoline arrangements with contact points 
supported by the dual pseudoline arrangement of $P$ minus its first level (3.2). Similarly, we observe that multitriangulations of a planar point set $P$ in convex position correspond to pseudoline arrangements with contact points supported by the dual pseudoline arrangement of $P$ minus its first $k$ levels (3.3).

This naturally yields to the definition of multipseudotriangulations in Sect. 4 . We study the primal of a multipseudotriangulation. We discuss some of its structural properties (4.2) which generalize the cases of pseudotriangulations and multitriangulations: number of edges, pointedness, crossing-freeness. We study in particular the faces of multipseudotriangulations (4.3) which naturally extend triangles in triangulations, pseudotriangles in pseudotriangulations, and stars in multitriangulations.

In Sect. 5, we compare multipseudotriangulations to iterated pseudotriangulations. We give an example of a 2-triangulation which is not a pseudotriangulation of a triangulation (5.1). We prove, however, that greedy multipseudotriangulations are iterated greedy pseudotriangulations (5.2), and we study flips in iterated pseudotriangulations (5.3).

Section 6 presents two further topics. The first one (6.1) is a pattern avoiding characterization of greedy multipseudotriangulations related to horizon trees. The second one (6.2) is a discussion on multipseudotriangulations of double pseudoline arrangements, which extend pseudotriangulations of convex bodies in the plane.

Finally, we discuss in Sect. 7 some related problems and open questions concerning in particular the primal of a multipseudotriangulation, the diameter and the polytopality of the graph of flips, and the number of multipseudotriangulations. Since the submission of this paper, some of these questions were partially answered in $[29,40,43]$ based on a framework similar to the material presented in this paper.

\section{Pseudoline Arrangements with the Same Support}

\subsection{Pseudoline Arrangements in the Möbius Strip}

Let $\mathcal{M}$ denote the Möbius strip (without boundary), defined as the quotient set of the plane $\mathbb{R}^{2}$ under the map $\tau: \mathbb{R}^{2} \rightarrow \mathbb{R}^{2},(x, y) \mapsto(x+\pi,-y)$. The induced canonical projection will be denoted by $\pi: \mathbb{R}^{2} \rightarrow \mathcal{M}$.

A pseudoline is the image $\lambda$ under the canonical projection $\pi$ of the graph $\{(x, f(x)) \mid x \in \mathbb{R}\}$ of a continuous and $\pi$-antiperiodic function $f: \mathbb{R} \rightarrow \mathbb{R}$ (that is, which satisfies $f(x+\pi)=-f(x)$ for all $x \in \mathbb{R})$. We say that $f$ represents the pseudoline $\lambda$.

When we consider two pseudolines, we always assume that they have a finite number of intersection points. Thus, these intersection points can only be either crossing points or contact points. See Fig. 3(a). Any two pseudolines always have an odd number of crossing points (in particular, at least one). When $\lambda$ and $\mu$ have exactly one crossing point, we denote it by $\lambda \wedge \mu$.

A pseudoline arrangement with contact points is a finite set $\Lambda$ of pseudolines such that any two of them have exactly one crossing point and possibly some contact 
Fig. 2 A pseudoline arrangement in the Möbius strip. Its contact points are represented by white circles

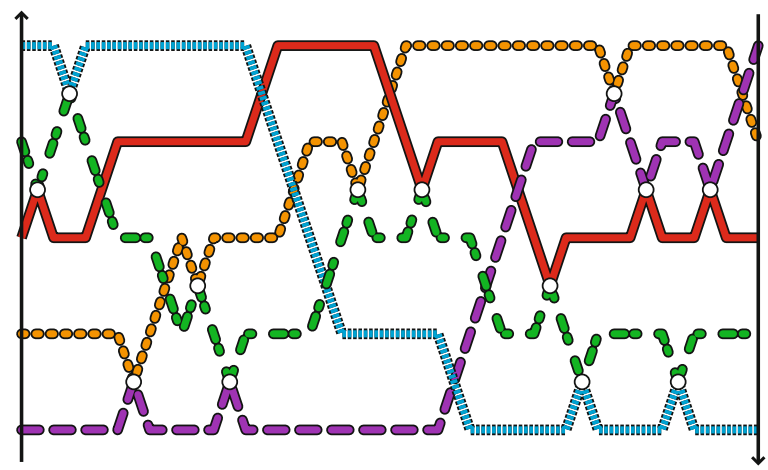

points. See Fig. 2. In this paper, we are only interested in simple arrangements, that is, where no three pseudolines meet in a common point. The support of $\Lambda$ is the union of its pseudolines. Observe that $\Lambda$ is completely determined by its support together with its set of contact points. The first level of $\Lambda$ is the external hull of the support of $\Lambda$, i.e. the boundary of the external face of the complement of the support of $\Lambda$. We define inductively the $k$ th level of $\Lambda$ as the external hull of the support of $\Lambda$ minus its first $k-1$ levels.

Remark 1 The usual definition of pseudoline arrangements does not allow contact points. In this paper, they play a crucial role since we are interested in all pseudoline arrangements which share a common support, and which only differ by their sets of contact points. To simplify the exposition, we omit throughout the paper to specify that we work with pseudoline arrangements with contact points.

Pseudoline arrangements are also classically defined on the projective plane rather than the Möbius strip. The projective plane is obtained from the Möbius strip by adding a point at infinity.

For more details on pseudoline arrangements, we refer to the broad literature on the topic $[6,13,14,24]$.

\subsection{Flip Graph and Greedy Pseudoline Arrangements}

\subsubsection{Flips}

We use the symbol $\triangle$ for the symmetric difference: $X \triangle Y:=(X \backslash Y) \cup(Y \backslash X)$. We refer to Fig. 3 for an illustration of the following lemma.

Lemma 2 Let $\Lambda$ be a pseudoline arrangement, $\mathcal{S}$ be its support, and $V$ be the set of its contact points. Let $v \in V$ be a contact point of two pseudolines of $\Lambda$, and $w$ denote their unique crossing point. Then $V \triangle\{v, w\}$ is also the set of contact points of a pseudoline arrangement $\Lambda^{\prime}$ supported by $\mathcal{S}$.

Proof Let $f$ and $g$ represent the two pseudolines $\lambda$ and $\mu$ of $\Lambda$ in contact at $v$. Let $x$ and $y$ be such that $v=\pi(x, f(x)), w=\pi(y, f(y))$ and $x<y<x+\pi$. We define two functions $f^{\prime}$ and $g^{\prime}$ by 
Fig. 3 (a) A pseudoline arrangement with one contact point $v$ and one crossing point $w$. (b) Flipping $v$ in the pseudoline arrangement of (a)

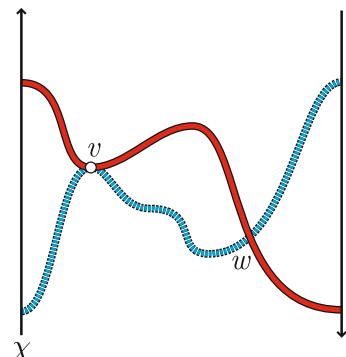

(a)

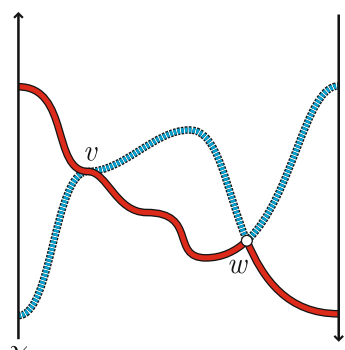

(b)

$$
f^{\prime}:=\left\{\begin{array}{ll}
f & \text { on }[x, y]+\mathbb{Z} \pi, \\
g & \text { otherwise }
\end{array} \quad \text { and } \quad g^{\prime}:= \begin{cases}g & \text { on }[x, y]+\mathbb{Z} \pi, \\
f & \text { otherwise. }\end{cases}\right.
$$

These two functions are continuous and $\pi$-antiperiodic, and thus define two pseudolines $\lambda^{\prime}$ and $\mu^{\prime}$. These two pseudolines have a contact point at $w$ and a unique crossing point at $v$, and they cross any pseudoline $v$ of $\Lambda \backslash\{\lambda, \mu\}$ exactly once (since $v$ either crosses both $\lambda$ and $\mu$ between $v$ and $w$, or crosses both $\lambda$ and $\mu$ between $w$ and $v$ ). Consequently, $\Lambda^{\prime}:=\Lambda \triangle\left\{\lambda, \mu, \lambda^{\prime}, \mu^{\prime}\right\}$ is a pseudoline arrangement, with support $\mathcal{S}$, and contact points $V \triangle\{v, w\}$.

Definition 3 Let $\Lambda$ be a pseudoline arrangement with support $\mathcal{S}$ and contact points $V$, let $v \in V$ be a contact point between two pseudolines of $\Lambda$ which cross at $w$, and let $\Lambda^{\prime}$ be the pseudoline arrangement with support $\mathcal{S}$ and contact points $V \triangle\{v, w\}$. We say that we obtain $\Lambda^{\prime}$ by flipping $v$ in $\Lambda$.

Note that the starting point of a flip is always a contact point. To this contact point corresponds precisely one crossing point. In contrast, it would be incorrect to try to flip a crossing point: the two pseudolines which cross at this point might have zero or many contact points.

Observe also that the pseudoline arrangements $\Lambda$ and $\Lambda^{\prime}$ are the only two pseudoline arrangements supported by $\mathcal{S}$ whose sets of contact points contain $V \backslash\{v\}$.

Definition 4 Let $\mathcal{S}$ be the support of a pseudoline arrangement. The flip graph of $\mathcal{S}$, denoted by $G(\mathcal{S})$, is the graph whose vertices are all the pseudoline arrangements supported by $\mathcal{S}$, and whose edges are flips between them.

In other words, there is an edge in the graph $G(\mathcal{S})$ between two pseudoline arrangements if and only if their sets of contact points differ by exactly two points.

Observe that the graph $G(\mathcal{S})$ is regular: there is one edge adjacent to a pseudoline arrangement $\Lambda$ supported by $\mathcal{S}$ for each contact point of $\Lambda$, and two pseudoline arrangements with the same support have the same number of contact points.

Example 5 The flip graph of the support of an arrangement of two pseudolines with $p$ contact points is the complete graph on $p+1$ vertices. 


\subsubsection{Acyclic Orientations}

Let $\mathcal{S}$ be the support of a pseudoline arrangement and $\overline{\mathcal{S}}$ denote its preimage under the projection $\pi$. We orient the graph $\overline{\mathcal{S}}$ along the abscissa increasing direction, and the graph $\mathcal{S}$ by projecting the orientations of the edges of $\overline{\mathcal{S}}$. We denote by $\preccurlyeq$ the induced partial order on the vertex set of $\overline{\mathcal{S}}$ (defined by $z \preccurlyeq z^{\prime}$ if there exists an oriented path on $\overline{\mathcal{S}}$ from $z$ to $z^{\prime}$ ).

A filter of $\overline{\mathcal{S}}$ is a proper set $F$ of vertices of $\overline{\mathcal{S}}$ such that $z \in F$ and $z \preccurlyeq z^{\prime}$ implies $z^{\prime} \in F$. The corresponding antichain is the set of all edges and faces of $\overline{\mathcal{S}}$ with one vertex in $F$ and one vertex not in $F$. This antichain has a linear structure, and thus, can be seen as the set of edges and faces that cross a vertical curve $\bar{\chi}$ of $\mathbb{R}^{2}$. The projection $\chi:=\pi(\bar{\chi})$ of such a curve is called a cut of $\mathcal{S}$. We see the fundamental domain located between the two curves $\bar{\chi}$ and $\tau(\bar{\chi})$ as the result of cutting the Möbius strip along the cut $\chi$. For example, we use such a cut to represent pseudoline arrangements in all figures of this paper. See for example Fig. 3.

The cut $\chi$ defines a partial order $\preccurlyeq \chi$ on the vertex set of $\mathcal{S}$ : for all vertices $v$ and $w$ of $\mathcal{S}$, we write $v \preccurlyeq \chi w$ if there is an oriented path in $\mathcal{S}$ which does not cross $\chi$. In other words, $v \preccurlyeq \chi w$ if $\bar{v} \preccurlyeq \bar{w}$, where $\bar{v}$ (resp. $\bar{w}$ ) denotes the unique preimage of $v$ (resp. $w$ ) between $\bar{\chi}$ and $\tau(\bar{\chi})$. For example, in the arrangements of Fig. 3, we have $v \prec_{\chi} w$.

Let $\Lambda$ be a pseudoline arrangement supported by $\mathcal{S}, v$ be a contact point between two pseudolines of $\Lambda$ and $w$ denote their crossing point. Since $v$ and $w$ lie on a same pseudoline on $\mathcal{S}$, they are comparable for $\preccurlyeq \chi$. We say that the flip of $v$ is $\chi$-increasing if $v \prec_{\chi} w$ and $\chi$-decreasing otherwise. For example, the flip from (a) to (b) in Fig. 3 is $\chi$-increasing. We denote by $G_{\chi}(\mathcal{S})$ the directed graph of $\chi$-increasing flips on pseudoline arrangements supported by $\mathcal{S}$.

Lemma 6 The directed graph $G_{\chi}(\mathcal{S})$ of $\chi$-increasing flips is acyclic.

Proof If $\Lambda$ and $\Lambda^{\prime}$ are two pseudoline arrangements supported by $\mathcal{S}$, we write $\Lambda \unlhd_{\chi} \Lambda^{\prime}$ if there exists a bijection $\phi$ between their sets of contact points such that $v \preccurlyeq \chi \phi(v)$ for any contact point $v$ of $\Lambda$. It is easy to see that this relation is a partial order on the vertices of $G_{\chi}(\mathcal{S})$. Since the edges of $G_{\chi}(\mathcal{S})$ are oriented according to $\star_{\chi}$, the graph $G_{\chi}(\mathcal{S})$ is acyclic.

Theorem 7 below states that this acyclic graph $G_{\chi}(\mathcal{S})$ has in fact a unique source, and thus is connected.

\subsubsection{Sorting Networks}

Let $n$ denote the number of pseudolines of the arrangements supported by $\mathcal{S}$ and $m \geq\left(\begin{array}{l}n \\ 2\end{array}\right)$ their number of intersection points (crossing points plus contact points). We consider a chain $F=F_{m} \supset F_{m-1} \supset \cdots \supset F_{1} \supset F_{0}=\tau(F)$ of filters of $\overline{\mathcal{S}}$ such that two consecutive of them $F_{i}$ and $F_{i+1}$ only differ by a single element: $\left\{\bar{v}_{i}\right\}:=F_{i+1} \backslash F_{i}$. This corresponds to a (backward) sweep $\chi=\chi_{0}, \chi_{1}, \ldots, \chi_{m}=\chi$ of the Möbius strip, where each cut $\chi_{i+1}$ is obtained from the cut $\chi_{i}$ by sweeping a 
Fig. 4 A (backward) sweep of the support of the pseudoline arrangement of Fig. 2

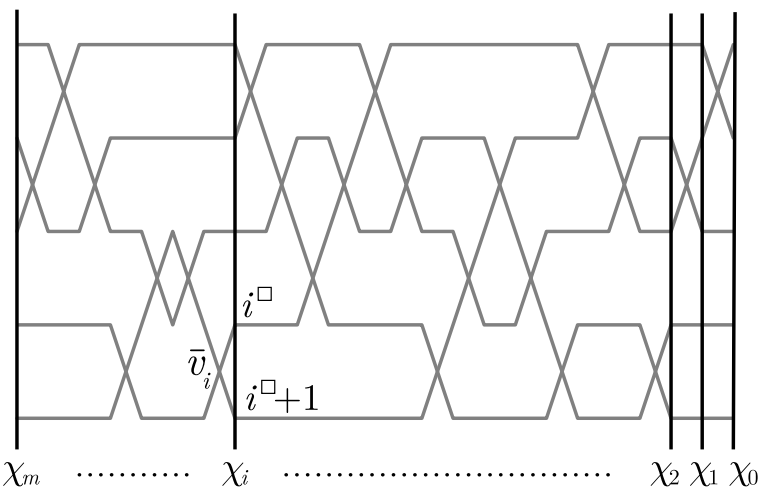

maximal vertex $v_{i}:=\pi\left(\bar{v}_{i}\right)$ of $\mathcal{S}$ (for the partial order $\preccurlyeq \chi$ ). For all $i$, let $e_{i}^{1}, e_{i}^{2}, \ldots, e_{i}^{n}$ denote the sequence of edges of $\overline{\mathcal{S}}$ with exactly one vertex in $F_{i}$, ordered from top to bottom, and let $i^{\square}$ be the index such that $\bar{v}_{i}$ is the common point of edges $e_{i}^{i^{\square}}$ and $e_{i}^{i^{\square}+1}$ (see Fig. 4).

Let $\Lambda:=\left\{\lambda_{1}, \ldots, \lambda_{n}\right\}$ be a pseudoline arrangement supported by $\mathcal{S}$. For all $i$, we denote by $\sigma_{i}^{\Lambda}$ the permutation of $\{1, \ldots, n\}$ whose $j$ th entry $\sigma_{i}^{\Lambda}(j)$ is the index of the pseudoline supporting $e_{i}^{j}$, i.e. such that $\pi\left(e_{i}^{j}\right) \subset \lambda_{\sigma_{i}^{\Lambda}(j)}$. Up to reindexing the pseudolines of $\Lambda$, we can assume that $\sigma_{0}^{\Lambda}$ is the inverted permutation $\sigma_{0}^{\Lambda}:=[n, n-1, \ldots, 2,1]$, and consequently that $\sigma_{m}^{\Lambda}$ is the identity permutation. Observe that for all $i$ :

(i) if $v_{i}$ is a contact point of $\Lambda$, then $\sigma_{i}^{\Lambda}=\sigma_{i+1}^{\Lambda}$;

(ii) otherwise, $\sigma_{i+1}^{\Lambda}$ is obtained from $\sigma_{i}^{\Lambda}$ by inverting its $i^{\square}$ th and $\left(i^{\square}+1\right)$ th entries.

The following theorem is illustrated on Fig. 5.

Theorem 7 The directed graph $G_{\chi}(\mathcal{S})$ has a unique source $\Gamma$, characterized by the property that the permutation $\sigma_{i+1}^{\Gamma}$ is obtained from $\sigma_{i}^{\Gamma}$ by sorting its $i^{\square}$ th and $\left(i^{\square}+1\right)$ th entries, for all $i$.

Proof If $\Gamma$ satisfies the above property, then it is obviously a source of the directed graph $G_{\chi}(\mathcal{S})$ : any flip of $\Gamma$ is $\chi$-increasing since two of its pseudolines cannot touch before they cross.

Assume conversely that $\Gamma$ is a source of $G_{\chi}(\mathcal{S})$. Let $a:=\sigma_{i}^{\Gamma}\left(i^{\square}\right)$ and $b:=\sigma_{i}^{\Gamma}\left(i^{\square}+1\right)$. We have two possible situations:

(i) If $a<b$, then the two pseudolines $\lambda_{a}$ and $\lambda_{b}$ of $\Gamma$ already cross before $v_{i}$. Consequently, $v_{i}$ is necessarily a contact point of $\Gamma$, which implies that $\sigma_{i+1}^{\Gamma}\left(i^{\square}\right)=a$ and $\sigma_{i+1}^{\Gamma}\left(i^{\square}+1\right)=b$.

(ii) If $a>b$, then the two pseudolines $\lambda_{a}$ and $\lambda_{b}$ of $\Gamma$ do not cross before $v_{i}$. Since $\Gamma$ is a source of $G_{\chi}(\mathcal{S}), v_{i}$ is necessarily a crossing point of $\Gamma$. Thus $\sigma_{i+1}^{\Gamma}\left(i^{\square}\right)=b$ and $\sigma_{i+1}^{\Gamma}(i \square+1)=a$. 
Fig. 5 The greedy pseudoline arrangement on the support of Fig. 2, obtained by sorting the permutation $[5,4,3,2,1]$. The result of each comparator is written after it (i.e. to its left since we sweep backwards)

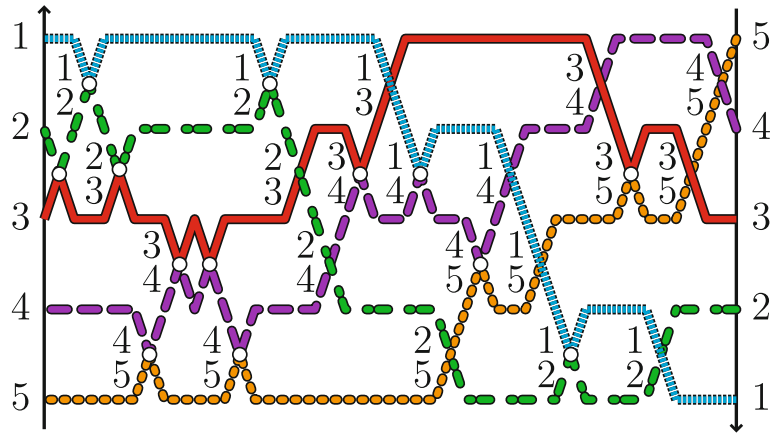

In both cases, $\sigma_{i+1}^{\Gamma}$ is obtained from $\sigma_{i}^{\Gamma}$ by sorting its $i^{\square}$ th and $\left(i^{\square}+1\right)$ th entries.

Corollary 8 The graphs of flips $G(\mathcal{S})$ and $G_{\chi}(\mathcal{S})$ are connected.

Definition 9 The unique source of the directed graph $G_{\chi}(\mathcal{S})$ is denoted by $\Gamma_{\chi}(\mathcal{S})$ and called the $\chi$-greedy pseudoline arrangement on $\mathcal{S}$.

Let us reformulate Theorem 7 in terms of sorting networks (see [23, Sect. 5.3.4] for a detailed presentation; see also [10]). Let $i<j$ be two integers. A comparator $[i: j]$ transforms a sequence of numbers $\left(a_{1}, \ldots, a_{n}\right)$ by sorting $\left(a_{i}, a_{j}\right)$, i.e. replacing $a_{i}$ by $\min \left(a_{i}, a_{j}\right)$ and $a_{j}$ by $\max \left(a_{i}, a_{j}\right)$. A comparator $[i: j]$ is primitive if $j=i+1$. A sorting network is a sequence of comparators that sorts any sequence $\left(a_{1}, \ldots, a_{n}\right)$.

The support $\mathcal{S}$ of an arrangement of $n$ pseudolines together with an arbitrary sweep $F_{m} \supset \cdots \supset F_{0}$ corresponds to the primitive sorting network $\left[1^{\square}: 1^{\square}+1\right], \ldots$, $\left[m^{\square}: m^{\square}+1\right]$ (see [24, Sect. 8]). Theorem 7 affirms that sorting the permutation $[n, n-1, \ldots, 2,1]$ according to this sorting network provides a pseudoline arrangement supported by $\mathcal{S}$, which depends only upon the support $\mathcal{S}$ and the filter $F_{0}$ (not on the total order given by the sweep).

\subsubsection{Greedy Set of Contact Points}

The following proposition provides an alternative construction of the greedy pseudoline arrangement $\Gamma_{\chi}(\mathcal{S})$ of the support $\mathcal{S}$.

Proposition 10 Let $v_{1}, \ldots, v_{q}$ be a sequence of vertices of $\mathcal{S}$ constructed recursively by choosing, as long as possible, a remaining vertex $v_{i}$ of $\mathcal{S}$ minimal (for the partial order $\preccurlyeq_{\chi}$ ) such that $\left\{v_{1}, \ldots, v_{i}\right\}$ is a subset of the set of contact points of a pseudoline arrangement supported by $\mathcal{S}$. Then the resulting set $\left\{v_{1}, \ldots, v_{q}\right\}$ is exactly the set of contact points of $\Gamma_{\chi}(\mathcal{S})$.

Proof First of all, $\left\{v_{1}, \ldots, v_{q}\right\}$ is by construction the set of contact points of a pseudoline arrangement $\Lambda$ supported by $\mathcal{S}$. If $\Lambda$ is not the (unique) source $\Gamma_{\chi}(\mathcal{S})$ of the oriented graph $G_{\chi}(\mathcal{S})$, then there is a contact point $v_{i}$ of $\Lambda$ whose flip is $\chi$-decreasing. Let $w$ denote the corresponding crossing point, and $\Lambda^{\prime}$ the pseudoline arrangement 
obtained from $\Lambda$ by flipping $v_{i}$. This implies that $\left\{v_{1}, \ldots, v_{i-1}, w\right\}$ is a subset of the set of contact points of $\Lambda^{\prime}$ and that $w \prec_{\chi} v_{i}$, which contradicts the minimality of $v_{i}$.

Essentially, this proposition affirms that we obtain the same pseudoline arrangement when:

(i) sweeping $\mathcal{S}$ decreasingly and place crossing points as long as possible; or

(ii) sweeping $\mathcal{S}$ increasingly and place contact points as long as possible.

\subsubsection{Constrained Flip Graph}

We now need to extend the previous results to constrained pseudoline arrangements on $\mathcal{S}$, in which we force a set $V$ of vertices of $\mathcal{S}$ to be contact points.

Theorem 11 Let $V$ be a subset of vertices of the support $\mathcal{S}$, and let $G_{\chi}(\mathcal{S} \mid V)$ be the subgraph of $G_{\chi}(\mathcal{S})$ induced by the pseudoline arrangements with support $\mathcal{S}$, whose set of contact points contains $V$. Then this directed graph $G_{\chi}(\mathcal{S} \mid V)$ is either empty or an acyclic connected graph with a unique source $\Gamma$ characterized by the property that for all $i$ :

(i) if $v_{i} \in V$, then $\sigma_{i+1}^{\Gamma}=\sigma_{i}^{\Gamma}$;

(ii) if $v_{i} \notin V$, then $\sigma_{i+1}^{\Gamma}$ is obtained from $\sigma_{i}^{\Gamma}$ by sorting its $i^{\square}$ th and $\left(i^{\square}+1\right)$ th entries.

Proof We transform our support $\mathcal{S}$ into another one $\mathcal{S}^{\prime}$ by opening all intersection points of $V$ (the local picture of this transformation is $\prec \rightarrow \asymp$ ). If $\mathcal{S}^{\prime}$ supports at least one pseudoline arrangement, we apply the result of Theorem 7: a pseudoline arrangement supported by $\mathcal{S}^{\prime}$ corresponds to a pseudoline arrangement with support $\mathcal{S}$ whose set of contact points contains $V$.

We denote by $\Gamma_{\chi}(\mathcal{S} \mid V)$ the unique source of the constrained flip graph $G_{\chi}(\mathcal{S} \mid V)$.

In terms of sorting networks, $\Gamma_{\chi}(\mathcal{S} \mid V)$ is the result of the sorting of the inverted permutation $[n, n-1, \ldots, 2,1]$ by the restricted primitive network $\left(\left[i^{\square}: i^{\square}+1\right]\right)_{i \in I}$, where $I:=\left\{i \mid v_{i} \notin V\right\}$.

Observe also that we can obtain, like in the previous subsection, the contact points of $\Gamma_{\chi}(\mathcal{S} \mid V)$ by an iterative procedure: we start from the set $V$ and add recursively a minimal (for the partial order $\preccurlyeq \chi$ ) remaining vertex $v_{i}$ of $\mathcal{S}$ such that $V \cup\left\{v_{1}, \ldots, v_{i}\right\}$ is a subset of the set of contact points of a pseudoline arrangement supported by $\mathcal{S}$. The vertex set produced by this procedure is the set of contact points of the $\chi$-greedy constrained pseudoline arrangement $\Gamma_{\chi}(\mathcal{S} \mid V)$.

\subsection{Greedy Flip Property and Enumeration}

\subsubsection{Greedy Flip Property}

We are now ready to state the greedy flip property (see Fig. 6) that says how to update the greedy pseudoline arrangement $\Gamma_{\chi}(\mathcal{S} \mid V)$ when either $\chi$ or $V$ are slightly perturbed. 

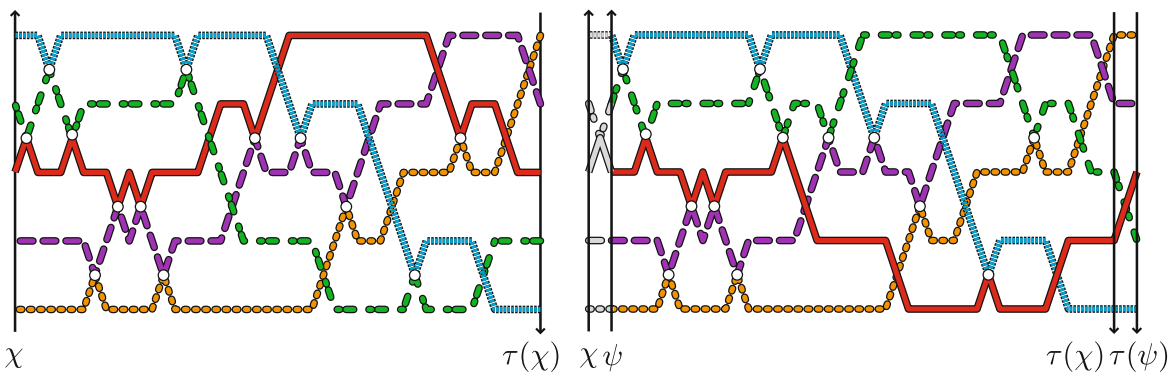

Fig. 6 The greedy flip property

Theorem 12 (Greedy flip property) Let $\mathcal{S}$ be the support of a pseudoline arrangement. Let $\chi$ be a cut of $\mathcal{S}, v$ be a minimal (for the order $\preccurlyeq \chi$ ) vertex of $\mathcal{S}$, and $\psi$ denote the cut obtained from $\chi$ by sweeping $v$. Let $V$ be a set of vertices of $\mathcal{S}$ (such that $G(\mathcal{S} \mid V)$ is not empty), and $W:=V \cup\{v\}$. Then:

(1) If $v$ is a contact point of $\Gamma_{\chi}(\mathcal{S} \mid V)$ which is not in $V$, then $\Gamma_{\psi}(\mathcal{S} \mid V)$ is obtained from $\Gamma_{\chi}(\mathcal{S} \mid V)$ by flipping $v$. Otherwise, $\Gamma_{\psi}(\mathcal{S} \mid V)=\Gamma_{\chi}(\mathcal{S} \mid V)$.

(2) If $v$ is a contact point of $\Gamma_{\chi}(\mathcal{S} \mid V)$, then $\Gamma_{\psi}(\mathcal{S} \mid W)=\Gamma_{\chi}(\mathcal{S} \mid V)$. Otherwise, $G(\mathcal{S} \mid W)$ is empty.

Proof We consider a sweep

$$
F_{m+1}=F \supset F_{m}=F^{\prime} \supset F_{m-1} \supset \cdots \supset F_{2} \supset F_{1}=\tau(F) \supset F_{0}=\tau\left(F^{\prime}\right)
$$

such that $F$ (resp. $F^{\prime}$ ) is a filter corresponding to the cut $\chi$ (resp. $\psi$ ). Define the points $\bar{v}_{i}:=F_{i+1} \backslash F_{i}$ and $v_{i}:=\pi\left(\bar{v}_{i}\right)$, and the index $i^{\square}$ as previously. Let $\sigma_{1}, \ldots, \sigma_{m+1}$ denote the sequence of permutations corresponding to $\Gamma_{\chi}(\mathcal{S} \mid V)$ on the sweep $F_{1} \subset \cdots \subset F_{m+1}$. In other words:

(i) $\sigma_{1}$ is the inverted permutation $[n, n-1, \ldots, 2,1]$;

(ii) if $v_{i} \in V$, then $\sigma_{i+1}=\sigma_{i}$;

(iii) otherwise, $\sigma_{i+1}$ is obtained from $\sigma_{i}$ by sorting its $i^{\square}$ th and $\left(i^{\square}+1\right)$ th entries.

Similarly, let $\rho_{0}, \ldots, \rho_{m}$ and $\omega_{0}, \ldots, \omega_{m}$ denote the sequences of permutations corresponding to $\Gamma_{\psi}(\mathcal{S} \mid V)$ and $\Gamma_{\psi}(\mathcal{S} \mid W)$, respectively, on the sweep $F_{0} \subset \cdots \subset F_{m}$.

Assume first that $v$ is a contact point of $\Gamma_{\chi}(\mathcal{S} \mid V)$, but is not in $V$. Let $j$ denote the integer such that $v_{j}$ is the crossing point of the two pseudolines of $\Gamma_{\chi}(\mathcal{S} \mid V)$ that are in contact at $v$. We claim that in this case $\Gamma_{\psi}(\mathcal{S} \mid V)$ is obtained from $\Gamma_{\chi}(\mathcal{S} \mid V)$ by flipping $v$, i.e. that:

(i) for all $1 \leq i \leq j, \rho_{i}$ is obtained from $\sigma_{i}$ by exchanging $m^{\square}$ and $m^{\square}+1$;

(ii) for all $j<i \leq m, \rho_{i}=\sigma_{i}$.

Indeed, $\rho_{1}$ is obtained by exchanging $m^{\square}$ and $m^{\square}+1$ in the sequence $\rho_{0}=[n, n-$ $1, \ldots, 2,1]=\sigma_{1}$ (since $m^{\square}$ and $m^{\square}+1$ are, respectively, the $\left(0^{\square}+1\right)$ th and $0^{\square}$ th entries of $\rho_{0}$ ). Then any comparison between two consecutive entries give the same result in $\rho_{i}$ and in $\sigma_{i}$, until $m^{\square}$ and $m^{\square}+1$ are compared again, i.e. until $i=j$. At 
this stage, $m^{\square}$ and $m^{\square}+1$ are already sorted in $\rho_{j}$ but not in $\sigma_{j}$. Consequently, we have to exchange $m^{\square}$ and $m^{\square}+1$ in $\sigma_{j}$ and not in $\rho_{j}$, and we obtain $\sigma_{j+1}=\rho_{j+1}$. After this, all the comparisons give the same result in $\rho_{i}$ and $\sigma_{i}$, and $\rho_{i}=\sigma_{i}$ for all $j<i \leq m$.

We prove similarly that:

- When $v$ is not a crossing point of $\Gamma_{\chi}(\mathcal{S} \mid V)$, or is in $V, \rho_{i}=\sigma_{i}$ for all $i \in[m]$, and $\Gamma_{\psi}(\mathcal{S} \mid V)=\Gamma_{\chi}(\mathcal{S} \mid V)$.

- When $v$ is a contact point of $\Gamma_{\chi}(\mathcal{S} \mid V), \omega_{i}=\sigma_{i}$ for all $i \in[m]$, and $\Gamma_{\psi}(\mathcal{S} \mid W)=$ $\Gamma_{\chi}(\mathcal{S} \mid V)$.

Finally, we prove that $G(\mathcal{S} \mid W)$ is empty when $v$ is not a contact point of $\Gamma_{\chi}(\mathcal{S} \mid V)$. For this, assume that $G(\mathcal{S} \mid W)$ is not empty, and consider the greedy arrangement $\Gamma=\Gamma_{\chi}(\mathcal{S} \mid W)$. The flip of any contact point of $\Gamma$ not in $W$ is $\chi$-increasing. Furthermore, since $v$ is a minimal element for $\preccurlyeq \chi$, the flip of $v$ is also $\chi$-increasing. Consequently, $\Gamma$ is a source in the graph $G_{\chi}(\mathcal{S} \mid V)$, which implies that $\Gamma_{\chi}(\mathcal{S} \mid V)=\Gamma$, and thus, $v$ is a contact point of $\Gamma_{\chi}(\mathcal{S} \mid V)$.

\subsubsection{Enumeration}

From the greedy flip property, we derive a binary tree structure on colored pseudoline arrangements supported by $\mathcal{S}$, whose left-pending leaves are precisely the pseudoline arrangements supported by $\mathcal{S}$. A pseudoline arrangement is colored if its contact points are colored in blue, green or red. Green and red contact points are considered to be fixed, while blue ones can be flipped.

Theorem 13 Let $\mathcal{T}$ be the binary tree on colored pseudoline arrangements supported by $\mathcal{S}$ defined as follows:

(i) The root of the tree is the $\chi$-greedy pseudoline arrangement on $\mathcal{S}$, entirely colored in blue.

(ii) Any node $\Lambda$ of $\mathcal{T}$ is a leaf of $\mathcal{T}$ if either it contains a green contact point or it only contains red contact points.

(iii) Otherwise, choose a minimal blue point $v$ of $\Lambda$. The right child of $\Lambda$ is obtained by flipping $v$ and coloring it in blue if the flip is $\chi$-increasing and in green if the flip is $\chi$-decreasing. The left child of $\Lambda$ is obtained by changing the color of $v$ into red.

Then the set of pseudoline arrangements supported by $\mathcal{S}$ is exactly the set of redcolored leafs of $\mathcal{T}$.

Proof The proof is similar to that of Theorem 9 in [7].

We define inductively a cut $\chi(\Lambda)$ for each node $\Lambda$ of $\mathcal{T}$ : the cut of the root is $\chi$, and for each node $\Lambda$ the cut of its children is obtained from $\chi$ by sweeping the contact point $v$. We also denote $V(\Lambda)$ the set of red contact points of $\Lambda$. With these notations, the greedy flip property (Theorem 12) ensures that $\Lambda=\Gamma_{\chi(\Lambda)}(\mathcal{S} \mid V(\Lambda))$, for each node $\Lambda$ of $\mathcal{T}$.

The fact that any red-colored leaf of $\mathcal{T}$ is a pseudoline arrangement supported by $\mathcal{S}$ is obvious. Reciprocally, let us prove that any pseudoline arrangement supported 
by $\mathcal{S}$ is a red leaf of $\mathcal{T}$. Let $\Lambda$ be a pseudoline arrangement supported by $\mathcal{S}$. We define inductively a path $\Lambda_{0}, \ldots, \Lambda_{p}$ in the tree $\mathcal{T}$ as follows: $\Lambda_{0}$ is the root of $\mathcal{T}$ and for all $i \geq 0, \Lambda_{i+1}$ is the left child of $\Lambda_{i}$ if the minimal blue contact point of $\Lambda_{i}$ is a contact point of $\Lambda$, and its right child otherwise (we stop when we reach a leaf). We claim that for all $0 \leq i \leq p$ :

- the set $V\left(\Lambda_{i}\right)$ is a subset of contact points of $\Lambda$;

- the contact points of $\Lambda$ not in $V\left(\Lambda_{i}\right)$ are not located between $\chi(\Lambda)$ and $\chi$;

from which we derive that $\Lambda=\Lambda_{p}$ is a red-colored leaf.

Visiting the tree $\mathcal{T}$ provides an algorithm to enumerate all pseudoline arrangements with a given support. In the next section, we will see the connection between this algorithm and the enumeration algorithm of [7] for pseudotriangulations of a point set.

Let us briefly discuss the complexity of this algorithm. We assume that the input of the algorithm is a pseudoline arrangement and we consider a flip as an elementary operation. Then this algorithm requires a polynomial running time per pseudoline arrangement supported by $\mathcal{S}$. As for many enumeration algorithms, the crucial point of this algorithm is that its working space is also polynomial (while the number of pseudoline arrangements supported by $\mathcal{S}$ is exponential).

\section{Dual Pseudoline Arrangements}

In this section, we prove that both the graph of flips on "(pointed) pseudotriangulations of a point set" and the graph of flips on "multitriangulations of a convex polygon" can be interpreted as graphs of flips on "pseudoline arrangements with a given support". This interpretation is based on the classical duality that we briefly recall in the first subsection, and leads to a natural definition of "multipseudotriangulations of a pseudoline arrangement" that we present in Sect. 4.

\subsection{Dual Pseudoline Arrangement of a Point Set}

To a given oriented line in the Euclidean plane, we associate its angle $\theta \in \mathbb{R} / 2 \pi \mathbb{Z}$ with the horizontal axis and its algebraic distance $d \in \mathbb{R}$ to the origin (i.e. the value of $\langle(-v, u) \mid$.$\rangle on the line, where (u, v)$ is its unitary direction vector). Since the same line oriented in the other direction gives an angle $\theta+\pi$ and a distance $-d$, this parametrization naturally associates a point of the Möbius strip $\mathcal{M}:=\mathbb{R}^{2} /(\theta, d) \sim(\theta+\pi,-d)$ to each line of the Euclidean plane. In other words, the line space of the Euclidean plane is (isomorphic to) the Möbius strip.

Via this parametrization, the set of lines passing through a point $p$ forms a pseudoline $p^{*}$. The pseudolines $p^{*}$ and $q^{*}$ dual to two distinct points $p$ and $q$ have a unique crossing point, namely the line $(p q)$. Thus, for a finite point set $P$ in the Euclidean plane, the set $P^{*}:=\left\{p^{*} \mid p \in P\right\}$ is a pseudoline arrangement without contact points (see Fig. 7). Again, we always assume that the point set $P$ is in general position (no three points lie in a same line), so that the arrangement $P^{*}$ is simple (no three pseudolines pass through the same point). 
Fig. 7 A point set $P$ in general position (a) and (a representation of) its dual arrangement $P^{*}(\mathbf{b})$

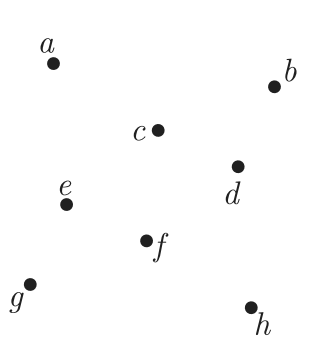

(a)

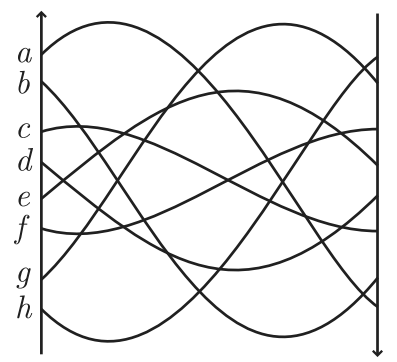

(b)

This elementary duality also holds for any topological plane (or $\mathbb{R}^{2}$-plane, see [39] for a definition), not only for the Euclidean plane $\mathbb{R}^{2}$. That is to say, the line space of a topological plane is (isomorphic to) the Möbius strip and the dual of a finite set of points in a topological plane is a pseudoline arrangement without contact points. Let us also recall that any pseudoline arrangement of the Möbius strip without contact points is the dual arrangement of a finite set of points in a certain topological plane [17]. Thus, in the rest of this paper, we deal with sets of points and their duals without restriction to the Euclidean plane.

\subsection{Dual Pseudoline Arrangement of a Pseudotriangulation}

We refer to [37] for a detailed survey on pseudotriangulations, and just recall here some basic definitions.

Definition 14 A pseudotriangle is a polygon $\Delta$ with only three convex vertices (the corners of $\Delta$ ), joined by three concave polygonal chains (Fig. 8). A line is said to be tangent to $\Delta$ if:

(i) either it passes through a corner of $\Delta$ and separates the two edges incident to it;

(ii) or it passes through a concave vertex of $\Delta$ and does not separate the two edges incident to it.

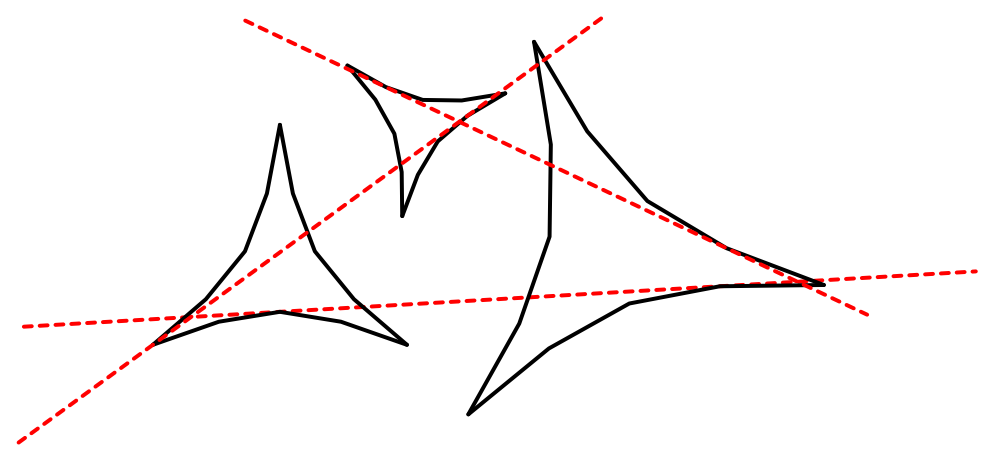

Fig. 8 Three pseudotriangles and their common tangents 


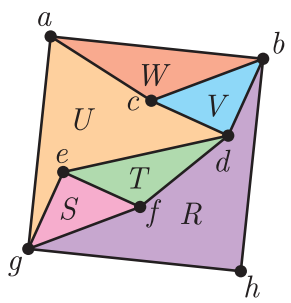

(a)

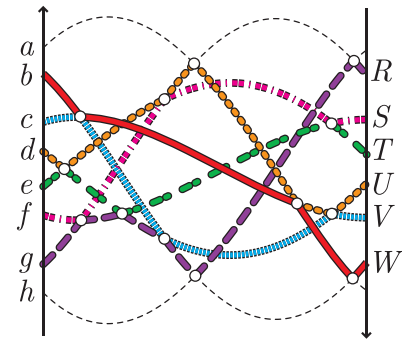

(b)

Fig. 9 (a) A pseudotriangulation $T$ of the point set $P$ of Fig. 7(a). (b) The dual arrangement $T^{*}$ of $T$, drawn on the dual arrangement $P^{*}$ of $P$ of Fig. 7(b). Each pseudoline of $T^{*}$ corresponds to a pseudotriangle of $T$; each contact point in $T^{*}$ corresponds to an edge in $T$; each crossing point in $T^{*}$ corresponds to a common tangent in $T$

A pseudotriangulation of a point set $P$ in general position is a set of edges of $P$ which decomposes the convex hull of $P$ into pseudotriangles. We moreover always assume that pseudotriangulations are pointed, meaning that there exists a line passing through any point $p \in P$ and defining a half-plane containing all the edges incident to $p$.

The results of this paper only concern pointed pseudotriangulations. Therefore we omit to always specify that pseudotriangulations are pointed. Historically, pseudotriangulations were introduced for families of smooth convex bodies [35] and were therefore automatically pointed. Pseudotriangulations of points, pointed or not, can be regarded as limits of pseudotriangulations of infinitesimally small convex bodies. Note that pointed pseudotriangulations are edge-minimal pseudotriangulations.

Under the pointedness assumption, any two pseudotriangles of a pseudotriangulation have a unique common tangent. This leads to the following observation (see Fig. 9):

Observation $15[32,33]$ Let $T$ be a pseudotriangulation of a point set $P$ in general position. Then:

(i) the set $\Delta^{*}$ of all tangents to a pseudotriangle $\Delta$ of $T$ is a pseudoline;

(ii) the dual pseudolines $\Delta_{1}^{*}, \Delta_{2}^{*}$ of any two pseudotriangles $\Delta_{1}, \Delta_{2}$ of $T$ have a unique crossing point (the unique common tangent to $\Delta_{1}$ and $\Delta_{2}$ ) and possibly a contact point (when $\Delta_{1}$ and $\Delta_{2}$ share a common edge);

(iii) the set $T^{*}:=\left\{\Delta^{*} \mid \Delta\right.$ pseudotriangle of $\left.T\right\}$ is a pseudoline arrangement (with contact points); and

(iv) $T^{*}$ is supported by $P^{*}$ minus its first level (see Fig. 9(b)).

In fact, this covering property characterizes pseudotriangulations:

Theorem 16 Let $P$ be a finite point set in general position in the plane, and $P^{* 1}$ denote the support of its dual pseudoline arrangement minus its first level. Then: 
Fig. 10 A flip in the pseudotriangulation of Fig. 9(a) and the corresponding flip in its dual pseudoline arrangement of Fig. 9(b)
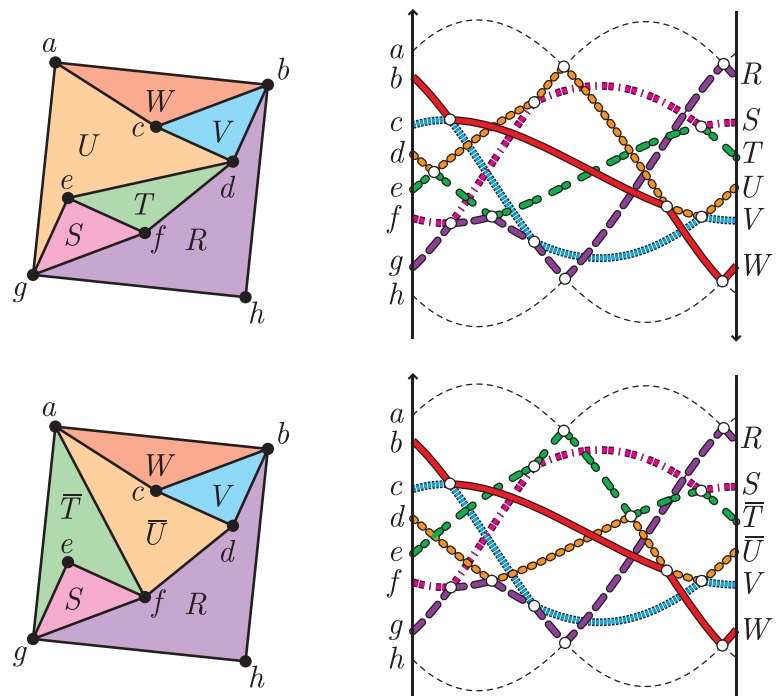

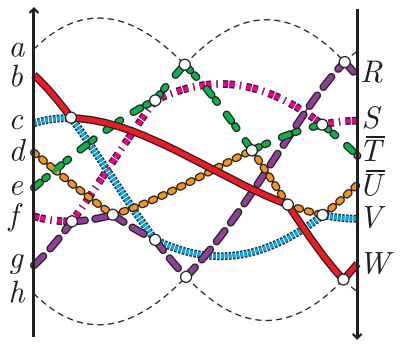

(i) The dual arrangement $T^{*}:=\left\{\Delta^{*} \mid \Delta\right.$ pseudotriangle of $\left.T\right\}$ of a pseudotriangulation $T$ of $P$ is supported by $P^{* 1}$.

(ii) The primal set of edges

$$
E:=\left\{[p, q] \mid p, q \in P, p^{*} \wedge q^{*} \text { contact point of } \Lambda\right\}
$$

of a pseudoline arrangement $\Lambda$ supported by $P^{* 1}$ is a pseudotriangulation of $P$.

In this section, we provide three proofs of part (ii) of this result. The first proof is based on flips. First, remember that there is also a simple flip operation on pseudotriangulations of $P$ : replacing any internal edge $e$ in a pseudotriangulation of $P$ by the common tangent of the two pseudotriangles containing $e$ produces a new pseudotriangulation of $P$. For example, Fig. 10 shows two pseudotriangulations of the point set of Fig. 7(a), related by a flip, together with their dual pseudoline arrangements. We denote by $G(P)$ the graph of flips on pseudotriangulations of $P$, whose vertices are pseudotriangulations of $P$ and whose edges are flips between them. In other words, there is an edge in $G(P)$ between two pseudotriangulations of $P$ if and only if their symmetric difference is reduced to a pair.

Proof 1 of Theorem 16(ii) The two notions of flips (the primal notion on pseudotriangulations of $P$ and the dual notion on pseudoline arrangements supported by $P^{* 1}$ ) coincide via duality: an internal edge $e$ of a pseudotriangulation $T$ of $P$ corresponds to a contact point $e^{*}$ of the dual pseudoline arrangement $T^{*}$; the two pseudotriangles $\Delta_{1}$ and $\Delta_{2}$ of $T$ containing $e$ correspond to the two pseudolines $\Delta_{1}^{*}$ and $\Delta_{2}^{*}$ of $T^{*}$ in contact at $e^{*}$; and the common tangent $f$ of $\Delta_{1}$ and $\Delta_{2}$ corresponds to the crossing point $f^{*}$ of $\Delta_{1}^{*}$ and $\Delta_{2}^{*}$.

Thus, the graph $G(P)$ is a subgraph of $G(\mathcal{S})$. Since both are connected and regular of degree $|P|-3$, they coincide. In particular, any pseudoline arrangement supported by $P^{* 1}$ is the dual of a pseudotriangulation of $P$. 
Fig. 11 (a) Four

points $p, q, r, s$ in convex position with the intersection $t$ of $[p, r]$ and $[q, s]$. (b) A point $s$ inside a triangle $p q r$ with the witness pseudoline $\ell_{\varepsilon}$

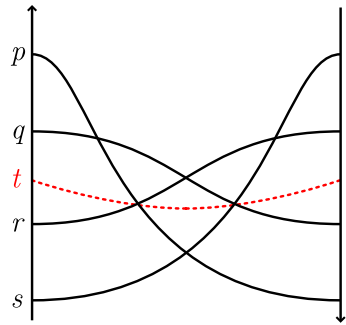

(a)

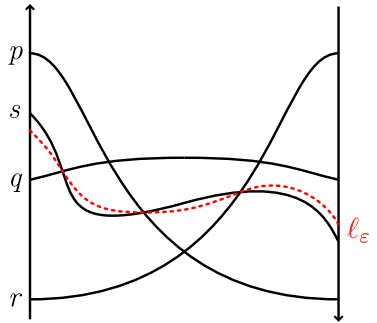

(b)

Remark 17 Observe that this duality matches our greedy pseudoline arrangement supported by $P^{* 1}$ with the greedy pseudotriangulation of [7]. In particular, the greedy flip property and the enumeration algorithm of Sect. 2.3 are generalizations of results in [7].

Our second proof of Theorem 16 is slightly longer but more direct, and it introduces a "witness method" that we will repeatedly use throughout this paper. It is based on the following characterization of pseudotriangulations:

Lemma 18 [42] A graph $T$ on $P$ is a pointed pseudotriangulation of $P$ if and only if it is crossing-free, pointed and has $2|P|-3$ edges.

Proof 2 of Theorem 16(ii) We check that $E$ is crossing-free, pointed and has $2|P|-3$ edges:

Cardinality. First, the number of edges of $E$ equals the difference between the number of crossing points of $P^{*}$ and of $\Lambda$ :

$$
|E|=\left(\begin{array}{c}
\left|P^{*}\right| \\
2
\end{array}\right)-\left(\begin{array}{c}
|\Lambda| \\
2
\end{array}\right)=\left(\begin{array}{c}
|P| \\
2
\end{array}\right)-\left(\begin{array}{c}
|P|-2 \\
2
\end{array}\right)=2|P|-3 .
$$

Crossing-free. Let $p, q, r, s$ be four points of $P$ in convex position. Let $t$ be the intersection of $[p, r]$ and $[q, s]$ (see Fig. 11(a)). We use the pseudoline $t^{*}$ as a witness to prove that $[p, r]$ and $[q, s]$ cannot both be in $E$. For this, we count crossings of $t^{*}$ with $P^{*}$ and $\Lambda$, respectively:

(i) Since $P$ is in general position, the point $t$ is not in $P$. Therefore $P^{*} \cup\left\{t^{*}\right\}=$ $(P \cup\{t\})^{*}$ is a (non-simple) pseudoline arrangement, and $t^{*} \operatorname{crosses} P^{*}$ exactly $|P|$ times.

(ii) Since $t^{*}$ is a pseudoline, it crosses each pseudoline of $\Lambda$ at least once. Thus, it crosses $\Lambda$ at least $|\Lambda|=|P|-2$ times.

(iii) For each of the points $p^{*} \wedge r^{*}$ and $q^{*} \wedge s^{*}$, replacing the crossing point by a contact point removes two crossings with $t^{*}$.

Thus, $[p, r]$ and $[q, s]$ cannot both be in $E$, and $E$ is crossing-free.

Pointed. Let $p, q, r, s$ be four points of $P$ such that $s$ lies inside the convex hull of $\{p, q, r\}$. We first construct a witness pseudoline (see Fig. 11(b)) that we use to 
prove that $[p, s],[q, s]$ and $[r, s]$ cannot all be in $E$. Let $f_{p}, f_{q}, f_{r}$ and $f_{s}$ represent $p^{*}, q^{*}, r^{*}$ and $s^{*}$, respectively. Let $x, y, z \in \mathbb{R}$ be such that $f_{p}(x)=f_{s}(x)$, $f_{q}(y)=f_{s}(y)$ and $f_{r}(z)=f_{s}(z)$. Let $g$ be a continuous and $\pi$-antiperiodic function vanishing exactly on $\{x, y, z\}+\mathbb{Z} \pi$ and changing sign each time it vanishes; say for example $g(t):=\sin (t-x) \sin (t-y) \sin (t-z)$. For all $\varepsilon>0$, we define the function $h_{\varepsilon}: \mathbb{R} \rightarrow \mathbb{R}$ by $h_{\varepsilon}(t)=f_{s}(t)+\varepsilon g(t)$. The function $h_{\varepsilon}$ is continuous and $\pi$-antiperiodic. The corresponding pseudoline $\ell_{\varepsilon}$ crosses $s^{*}$ three times. It is also easy to see that if $\varepsilon$ is sufficiently small, then $\ell_{\varepsilon}$ crosses the pseudolines of $(P \backslash\{s\})^{*}$ exactly as $s^{*}$ does (see Fig. 11(b)). For such a small $\varepsilon$, we count the crossings of $\ell_{\varepsilon}$ with $P^{*}$ and $\Lambda$, respectively:

(i) $\ell_{\varepsilon}$ crosses $P^{*}$ exactly $|P|+2$ times (it crosses $s^{*}$ three times and any other pseudoline of $P^{*}$ exactly once).

(ii) Since $\ell_{\varepsilon}$ is a pseudoline, it crosses $\Lambda$ at least $|\Lambda|=|P|-2$ times.

(iii) For each of the points $p^{*} \wedge s^{*}, q^{*} \wedge s^{*}$ and $r^{*} \wedge s^{*}$, replacing the crossing point by a contact point removes two crossings with $\ell_{\varepsilon}$.

Thus, $[p, r],[q, s]$ and $[r, s]$ cannot all be in $E$, and $E$ is pointed.

Observe that once we know that $E$ is crossing-free, we could also argue its pointedness observing that the pseudolines of $\Lambda$ would cover the dual pseudoline of a non-pointed vertex twice.

Our third proof of Theorem 16 focusses on pseudotriangles. For every pseudoline $\lambda$ of the pseudoline arrangement $\Lambda$, we denote by

$$
S(\lambda):=\left\{[p, q] \mid p, q \in P, p^{*} \wedge q^{*} \text { contact point of } \lambda\right\}
$$

the polygonal cycle formed by the edges primal to the contact points of $\lambda$. For any point $q$ in the plane, we denote by $\sigma_{\lambda}(q)$ the winding number of $S(\lambda)$ around $q$, i.e. the number of rounds made by $S(\lambda)$ around the point $q$.

Proof 3 of Theorem 16(ii) Consider a point $q$ inside the convex hull of our point set $P$, and such that $P \cup\{q\}$ be in general position. Hence, its dual pseudoline $q^{*}$ has exactly $|P|$ crossings with $P^{*}$, none of which are on the first level of $P^{*}$. For any pseudoline $\lambda \in \Lambda$, let $\tau_{\lambda}(q)$ denote the number of intersection points between $q^{*}$ and $\lambda$ (that is, the number of tangents to $S(\lambda)$ passing through $q$ ). Then we have $\sigma_{\lambda}(q)=\left(\tau_{\lambda}(q)-1\right) / 2$ and

$$
\begin{aligned}
|P| & =\left|q^{*} \cap P^{* 1}\right|=\sum_{\lambda \in \Lambda} \tau_{\lambda}(q)=|\Lambda|+2 \sum_{\lambda \in \Lambda} \sigma_{\lambda}(q) \\
& =|P|-2+2 \sum_{\lambda \in \Lambda} \sigma_{\lambda}(q) .
\end{aligned}
$$

Consequently, $\sum_{\lambda \in \Lambda} \sigma_{\lambda}(q)=1$. Hence $\sigma_{\lambda}(q)=0$ for all $\lambda \in \Lambda$, except for precisely one pseudoline $\mu \in \Lambda$ which satisfies $\sigma_{\mu}(q)=1$.

As a consequence, all polygons $S(\lambda)$ for $\lambda \in \Lambda$ are pseudotriangles (otherwise, we would have points such that $\sigma_{\lambda}(q)>1$ for some $\lambda$ ) and they cover the convex hull of $P$. Consequently, these $|P|-2$ pseudotriangles form a pointed pseudotriangulation of $P$. 
Fig. 12 A 2-triangulation of the octagon

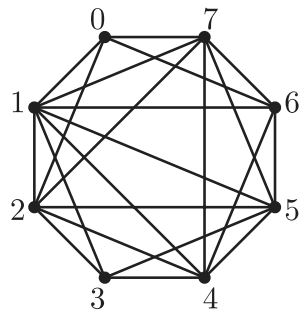

\subsection{Dual Pseudoline Arrangement of a Multitriangulation}

Let $C_{n}$ denote the set of vertices of the convex regular $n$-gon. We are interested in the following generalization of triangulations, introduced by Capoyleas and Pach [9] in the context of extremal theory for geometric graphs (see Fig. 12).

Definition 19 For $\ell \in \mathbb{N}$, an $\ell$-crossing is a set of $\ell$ mutually crossing edges of $C_{n}$. A $k$-triangulation of the $n$-gon is a maximal set of edges of $C_{n}$ with no $(k+1)$-crossing.

Observe that an edge of $C_{n}$ can be involved in a $(k+1)$-crossing only if there remain at least $k$ vertices on each side. Such an edge is called $k$-relevant. An edge with exactly (resp. strictly less than) $k-1$ vertices on one side is a $k$-boundary edge (resp. a $k$-irrelevant edge). By maximality, every $k$-triangulation consists of all the $n k k$-irrelevant plus $k$-boundary edges and some $k$-relevant edges.

In [28], the triangles and their bisectors are generalized for $k$-triangulations as follows (see Fig. 13):

Definition 20 [28] A $k$-star is a star polygon of type $\{2 k+1 / k\}$, that is, a set of edges of the form $\left\{s_{j} s_{j+k} \mid j \in \mathbb{Z}_{2 k+1}\right\}$, where $s_{0}, s_{1}, \ldots, s_{2 k}$ are cyclically ordered around the unit circle. A (strict) bisector of a $k$-star is a (strict) bisector of one of its angles $s_{j-k} s_{j} s_{j+k}$.

As for $k=1$, where triangles provide a powerful tool to study triangulations, $k$-stars are useful to understand $k$-triangulations. In the following theorem, we point out five properties of stars proved in [28]. Figures 13 and 14 illustrate these results on the 2-triangulation of Fig. 12.
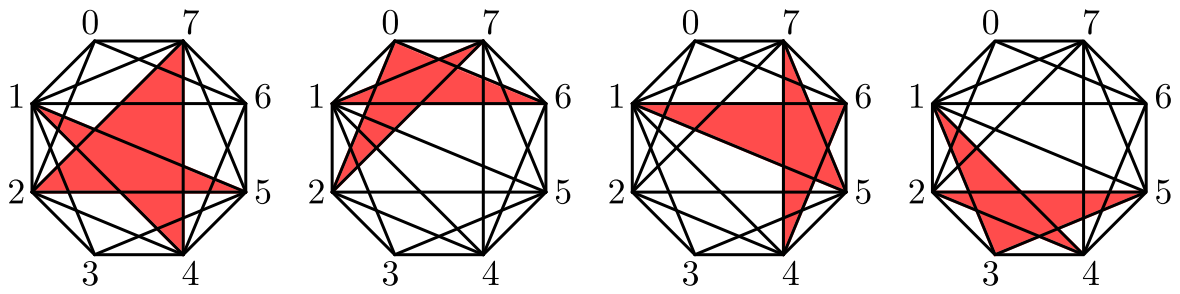

Fig. 13 The four 2-stars of the 2-triangulation of Fig. 12 
Fig. 14 A flip in the

2-triangulation of Fig. 12
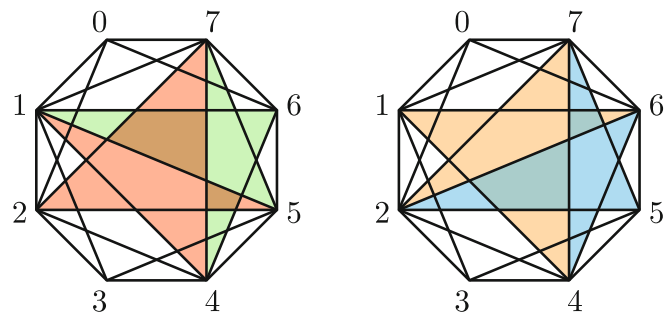

Theorem 21 [28] Let $T$ be a $k$-triangulation of the $n$-gon. Then

(i) $T$ contains exactly $n-2 k k$-stars and $k(n-2 k-1) k$-relevant edges.

(ii) Each edge of $T$ belongs to zero, one, or two $k$-stars, depending on whether it is $k$-irrelevant, $k$-boundary, or $k$-relevant.

(iii) Every pair of $k$-stars of $T$ has a unique common strict bisector.

(iv) Flipping any $k$-relevant edge e of $T$ into the common strict bisector $f$ of the two $k$-stars containing e produces a new $k$-triangulation $T \triangle\{e, f\}$ of the $n$-gon. $T$ and $T \triangle\{e, f\}$ are the only two $k$-triangulations of the $n$-gon containing $T \backslash\{e\}$.

(v) The flip graph $G_{n, k}$ on $k$-triangulations of the $n$-gon is connected and regular of degree $k(n-2 k-1)$.

Similarly to Observation 15 , we can interpret these properties of the stars of the multitriangulations in the dual space (see Fig. 15):

Observation 22 Let $T$ be a $k$-triangulation of a convex $n$-gon. Then:

(i) the set $S^{*}$ of all bisectors of a $k$-star $S$ of $T$ is a pseudoline of the Möbius strip;

(ii) the dual pseudolines $S_{1}^{*}, S_{2}^{*}$ of any two $k$-stars $S_{1}, \Delta_{2}$ of $T$ have a unique crossing point (the unique common strict bisector of $S_{1}$ and $S_{2}$ ) and possibly some contact points (when $S_{1}$ and $S_{2}$ share common edges);

(iii) the set $T^{*}:=\left\{S^{*} \mid S k\right.$-star of $\left.T\right\}$ of dual pseudolines of $k$-stars of $T$ is a pseudoline arrangement (with contact points); and

(iv) $T^{*}$ is supported by the dual pseudoline arrangement $C_{n}^{*}$ of $C_{n}$ minus its first $k$ levels (see Fig. 15(b)).

Fig. 15 A 2-triangulation $T$ of the octagon (a) and its dual pseudoline arrangement $T^{*}(\mathbf{b})$. Each thick pseudoline of $T^{*}$ corresponds to a 2-star of $T$; each contact point in $T^{*}$ corresponds to an edge in $T$; each crossing point in $T^{*}$ corresponds to a common bisector in $T$

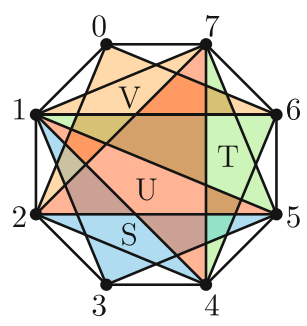

(a)

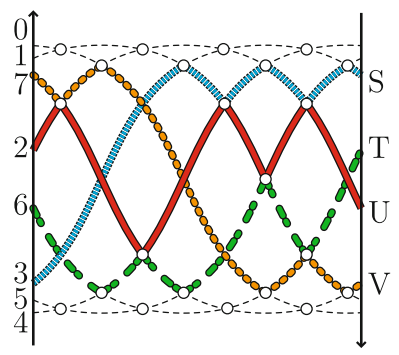

(b) 
Again, it turns out that this observation provides a characterization of multitriangulations of a convex polygon:

Theorem 23 Let $C_{n}$ denote the set of vertices of a convex $n$-gon, and $C_{n}^{* k}$ denote the support of its dual pseudoline arrangement minus its first $k$ levels. Then:

(i) The dual pseudoline arrangement $T^{*}:=\left\{S^{*} \mid S k\right.$-star of $\left.T\right\}$ of a $k$-triangulation $T$ of the $n$-gon is supported by $C_{n}^{* k}$.

(ii) The primal set of edges

$$
E:=\left\{[p, q] \mid p, q \in C_{n}, p^{*} \wedge q^{*} \text { contact point of } \Lambda\right\}
$$

of a pseudoline arrangement $\Lambda$ supported by $C_{n}^{* k}$ is a $k$-triangulation of the n-gon.

We provide two proofs of this theorem.

Proof 1 of Theorem 23(ii) The two notions of flips (the primal notion on $k$-triangulations of the $n$-gon and the dual notion on pseudoline arrangements supported by $C_{n}^{* k}$ ) coincide. Thus, the flip graph $G_{n, k}$ on $k$-triangulations of the $n$-gon is a subgraph of $G\left(C_{n}^{* k}\right)$. Since they are both connected and regular of degree $k(n-2 k-1)$, these two graphs coincide. In particular, any pseudoline arrangement supported by $C_{n}^{* k}$ is the dual of a $k$-triangulation of the $n$-gon.

Proof 2 of Theorem 23(ii) We follow the method of our second proof of Theorem 16(ii). Since $E$ has the right number of edges (namely $k(2 n-2 k-1)$ ), we only have to prove that it is $(k+1)$-crossing-free. We consider $2 k+2$ points $p_{0}, \ldots, p_{k}$, $q_{0}, \ldots, q_{k}$ cyclically ordered around the unit circle. Since the definition of crossing (and thus, of $\ell$-crossing) is purely combinatorial, i.e. depends only on the cyclic order of the points and not on their exact positions, we can move all the vertices of our $n$-gon on the unit circle while preserving their cyclic order. In particular, we can assume that the lines $\left(p_{i} q_{i}\right)_{i \in\{0, \ldots, k\}}$ all contain a common point $t$. Its dual pseudoline $t^{*}$ crosses $C_{n}^{*}$ exactly $n$ times and $\Lambda$ at least $|\Lambda|=n-2 k$ times. Furthermore, for any point $p_{i}^{*} \wedge q_{i}^{*}$, replacing the crossing point by a contact point removes two crossings with $t^{*}$. Thus, the pseudoline $t^{*}$ provides a witness which proves that the edges $\left[p_{i}, q_{i}\right], i \in\{0, \ldots, k\}$, cannot be all in $E$, and thus ensures that $E$ is $(k+1)$-crossing-free.

\section{Multipseudotriangulations}

Motivated by Theorems 16 and 23, we define in terms of pseudoline arrangements a natural generalization of both pseudotriangulations and multitriangulations. We then study elementary properties of the corresponding set of edges in the primal space. 


\subsection{Definition}

We consider the following generalizations of both pseudotriangulations and multitriangulations:

Definition 24 Let $L$ be a pseudoline arrangement supported by $\mathcal{S}$. Define its $k$-kernel $\mathcal{S}^{k}$ to be its support minus its first $k$ levels (which are the iterated external hulls of $\mathcal{S}$ ). Denote by $V^{k}$ the set of contact points of $L$ in $\mathcal{S}^{k}$. A $k$-pseudotriangulation of $L$ is a pseudoline arrangement whose support is $\mathcal{S}^{k}$ and whose set of contact points contains $V^{k}$.

Pseudotriangulations of a point set $P$ correspond via duality to 1-pseudotriangulations of the dual pseudoline arrangement $P^{*}$. Similarly, $k$-triangulations of the $n$-gon correspond to $k$-pseudotriangulations of the pseudoline arrangement $C_{n}^{*}$ in convex position. If $L$ is a pseudoline arrangement with no contact point, then any pseudoline arrangement supported by $\mathcal{S}^{k}$ is a $k$-pseudotriangulation of $L$. In general, the condition that the contact points of $L$ in its $k$-kernel should be contact points of any $k$-pseudotriangulation of $L$ is a natural assumption for iterating multipseudotriangulations (see Sect. 5).

Let $\Lambda$ be a $k$-pseudotriangulation of $L$. We denote by $V(\Lambda)$ the union of the set of contact points of $\Lambda$ with the set of intersection points of the first $k$ levels of $L$. In other words, $V(\Lambda)$ is the set of intersection points of $L$ which are not crossing points of $\Lambda$. As for pseudoline arrangements, the set $V(\Lambda)$ completely determines $\Lambda$.

Flips for multipseudotriangulations are defined as in Lemma 2, with the restriction that the contact points in $V^{k}$ cannot be flipped. In other words, the flip graph on $k$-pseudotriangulations of $L$ is exactly the graph $G\left(\mathcal{S}^{k} \mid V^{k}\right)$. Section 2 asserts that the graph of flips is regular and connected, and provides an enumeration algorithm for multipseudotriangulations of $L$.

Let $\chi$ be a cut of (the support of) $L$. It is also a cut of the $k$-kernel $\mathcal{S}^{k}$ of $L$. A particularly interesting example of $k$-pseudotriangulation of $L$ is the source of the graph of $\chi$-increasing flips on $k$-pseudotriangulations of $L$ (see Fig. 16 for an illustration):

Definition 25 The $\chi$-greedy k-pseudotriangulation of $L$, denoted $\Gamma_{\chi}^{k}(L)$, is the greedy pseudoline arrangement $\Gamma_{\chi}\left(\mathcal{S}^{k} \mid V^{k}\right)$.

\subsection{Pointedness and Crossings}

Let $P$ be a point set in general position. Let $\Lambda$ be a $k$-pseudotriangulation of $P^{*}$ and $V(\Lambda)$ be the set of crossing points of $P^{*}$ which are not crossing points of $\Lambda$. We call primal of $\Lambda$ the set

$$
E:=\left\{[p, q] \mid p, q \in P, p^{*} \wedge q^{*} \in V(\Lambda)\right\}
$$

of edges of $P$ primal to $V(\Lambda)$ (see Fig. 17). Here, we discuss general properties of primals of multipseudotriangulations. We start with elementary properties that we 
Fig. 16 The $\chi$-greedy

1 -pseudotriangulation and the $\chi$-greedy 2-pseudotriangulation of the pseudoline arrangement of Fig. 7(b)
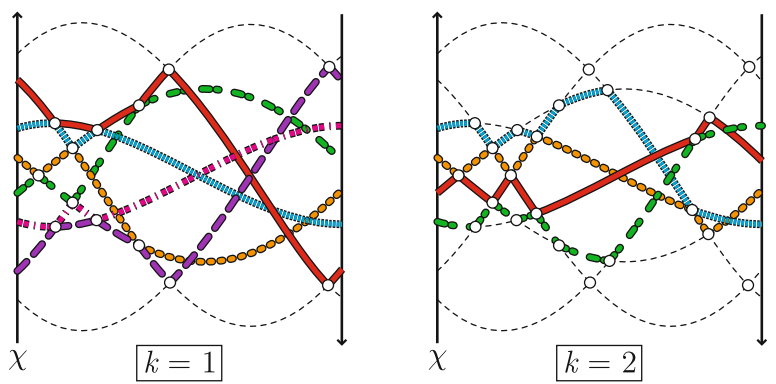

Fig. 17 The primal set of edges (a) of a

2-pseudotriangulation (b) of the dual pseudoline of the point set of Fig. 7(a)

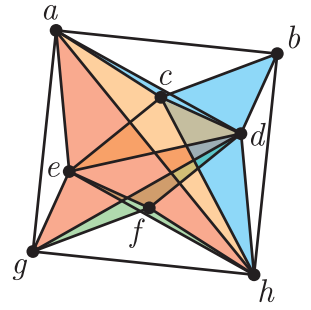

(a)

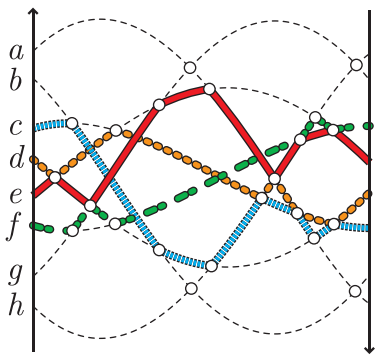

(b)

already observed for the special cases of pseudotriangulations and multitriangulations in the proofs of Theorems 16 and 23:

Lemma 26 The primal $E$ of $\Lambda$ has $k(2|P|-2 k-1)$ edges.

Proof The number of edges of $E$ is the difference between the number of crossing points in the pseudoline arrangements $P^{*}$ and $\Lambda$ :

$$
|E|=\left(\begin{array}{c}
\left|P^{*}\right| \\
2
\end{array}\right)-\left(\begin{array}{c}
|\Lambda| \\
2
\end{array}\right)=\left(\begin{array}{c}
|P| \\
2
\end{array}\right)-\left(\begin{array}{c}
|P|-2 k \\
2
\end{array}\right)=k(2|P|-2 k-1) .
$$

We now discuss pointedness of $E$. We call $k$-alternation any set $\left\{f_{i} \mid i \in \mathbb{Z}_{2 k+1}\right\}$ of $2 k+1$ edges all incident to a common vertex and whose cyclic order around it is given by

$$
f_{0} \prec \bar{f}_{1+k} \prec f_{1} \prec \bar{f}_{2+k} \prec \cdots \prec f_{2 k} \prec \bar{f}_{k} \prec f_{0},
$$

where $\bar{f}_{i}$ denotes the opposite direction of the edge $f_{i}$.

Lemma 27 The primal $E$ of $\Lambda$ cannot contain a $k$-alternation.

Proof We simply mimic the proof of pointedness in Theorem 16. Let $p_{0}, \ldots, p_{2 k}$ and $q$ be $2 k+2$ points of $P$ such that $F:=\left\{\left[p_{i}, q\right] \mid i \in \mathbb{Z}_{2 k+1}\right\}$ is a $k$-alternation. We prove that $F$ cannot be a subset of $E$ by constructing a witness pseudoline $\ell$ that separates all the crossing points $p_{i}^{*} \wedge q^{*}$ corresponding to $F$, while crossing $q^{*}$ exactly $2 k+1$ times and the other pseudolines of $P^{*}$ exactly as $q^{*}$ does. (We skip 


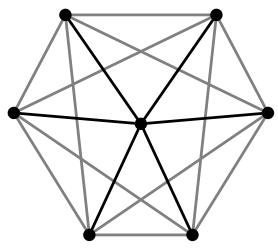

(a)

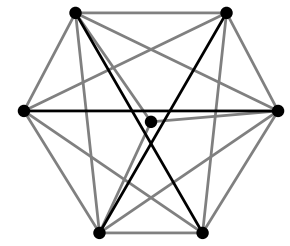

(b)

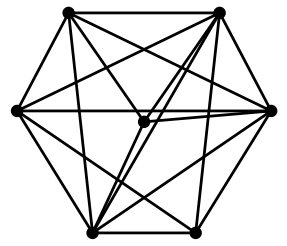

(c)

Fig. 18 (a) A non-2-pointed (but 2-alternation-free) 2-pseudotriangulation. (b) A 2-pseudotriangulation containing a 3-crossing. (c) A 3-crossing-free 2-alternation-free set not contained in a 2-pseudotriangulation

the precise construction, since it is exactly the same as in the proof of Theorem 16.) Counting the crossings of $\ell$ with $P^{*}$ and $\Lambda$, we obtain:

(i) $\ell$ crosses $P^{*}$ exactly $|P|+2 k$ times;

(ii) $\ell$ crosses $\Lambda$ at least $|\Lambda|=|P|-2 k$ times;

(iii) for each of the points $p_{i}^{*} \wedge q^{*}$, replacing the crossing point by a contact point removes two crossings with $\ell$.

Thus the edges $\left[p_{i}, q\right]$ cannot all be contained in $E$.

Remark 28 Observe that a set of edges is pointed if and only if it is 1-alternation-free. In contrast, we want to observe the difference between $k$-alternation-freeness and the following natural notion of $k$-pointedness: we say that a set $F$ of edges with vertices in $P$ is $k$-pointed if for all $p$ in $P$, there exists a line which passes through $p$ and defines a half-plane that contains at most $k-1$ segments of $F$ adjacent to $p$. Observe that a $k$-pointed set is automatically $k$-alternation-free but that the converse statement does not hold (see Fig. 18(a)).

Finally, contrarily to pseudotriangulations $(k=1)$ and multitriangulations (convex position), the condition of avoiding $(k+1)$-crossings does not hold for $k$-pseudotriangulations in general:

Remark 29 There exist $k$-pseudotriangulations with $(k+1)$-crossings (see Fig. 18(b)) as well as $(k+1)$-crossing-free $k$-alternation-free sets of edges that are not subsets of $k$-pseudotriangulations (see Fig. 18(c)).

\subsection{Stars in Multipseudotriangulations}

To complete our understanding of the primal of multipseudotriangulations, we need to generalize pseudotriangles of pseudotriangulations and $k$-stars of $k$-triangulations: both pseudotriangles and $k$-stars correspond to pseudolines of the covering pseudoline arrangement.

We keep the notations of the previous section: $P$ is a point set in general position, $\Lambda$ is a $k$-pseudotriangulation of $P^{*}$ and $E$ is the primal set of edges of $\Lambda$. 
Fig. 19 (a) Winding number of a star. (b) 2-depth in the point set of Fig. 7(a)

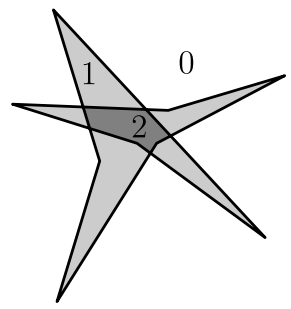

(a)

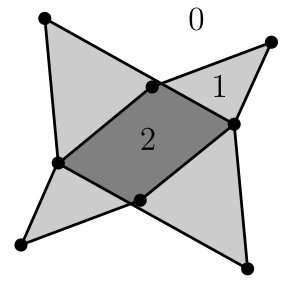

(b)

Definition 30 We call star of a pseudoline $\lambda \in \Lambda$ the set of edges

$$
S(\lambda):=\left\{[p, q] \mid p, q \in P, p^{*} \wedge q^{*} \text { contact point of } \lambda\right\}
$$

primal to the contact points of $\lambda$.

Lemma 31 For any $\lambda \in \Lambda$, the star $S(\lambda)$ is non-empty.

Proof We have to prove that any pseudoline $\lambda$ of $\Lambda$ supports at least one contact point. If it is not the case, then $\lambda$ is also a pseudoline of $P^{*}$, and all the $|P|-1$ crossing points of $\lambda$ with $P^{*} \backslash\{\lambda\}$ should be crossing points of $\lambda$ with the arrangement $\Lambda \backslash\{\lambda\}$. This is impossible since $|\Lambda \backslash\{\lambda\}|=|P|-2 k-1$.

Similarly to the case of $k$-triangulations of the $n$-gon, we say that an edge $[p, q]$ of $E$ is a $k$-relevant (resp. $k$-boundary, resp. $k$-irrelevant) edge if there remain strictly more than (resp. exactly, resp. strictly less than) $k-1$ points of $P$ on each side (resp. one side) of the line $(p q)$. In other words, $p^{*} \wedge q^{*}$ is located in the $k$-kernel (resp. in the intersection of the $k$ th level and the $k$-kernel, resp. in the first $k$ levels) of the pseudoline arrangement $P^{*}$. Thus, the edge $[p, q]$ is contained in 2 (resp. 1 , resp. 0) stars of $\Lambda$.

The edges of a star $S(\lambda)$ are cyclically ordered by the order of their dual contact points on $\lambda$, and thus $S(\lambda)$ forms a (not-necessarily simple) polygonal cycle. For any point $q$ in the plane, let $\sigma_{\lambda}(q)$ denote the winding number of $S(\lambda)$ around $q$, that is, the number of rounds made by $S(\lambda)$ around the point $q$ (see Fig. 19(a)). For example, the winding number of a point in the external face is 0 .

We call $k$-depth of a point $q$ the number $\delta^{k}(q)$ of $k$-boundary edges of $P$ crossed by any (generic continuous) path from $q$ to the external face, counted positively when passing from the "big" side (the one containing at least $k$ vertices of $P$ ) to the "small side" (the one containing $k-1$ vertices of $P$ ), and negatively otherwise (see Fig. 19(b)). That this number is independent from the path can be seen by mutation. For example, $\delta^{1}(q)$ is 1 if $q$ is in the convex hull of $P$ and 0 otherwise.

Proposition 32 Any point $q$ of the plane is covered $\delta^{k}(q)$ times by the stars $S(\lambda)$, $\lambda \in \Lambda$, of the $k$-pseudotriangulation $\Lambda$ of $P^{*}$ :

$$
\delta^{k}(q)=\sum_{\lambda \in \Lambda} \sigma_{\ell}(q) .
$$


The proposition is intuitively clear: let us walk on a continuous path from the external face to the point $q$. Initially, the winding numbers of all stars of $\Lambda$ are zero (we start outside all stars of $\Lambda$ ). Then each time we cross an edge $e$ :

(i) If $e$ is $k$-irrelevant, it is not contained in any star of $\Lambda$, and we do not change the winding numbers of the stars of $\Lambda$.

(ii) If $e$ is a $k$-boundary edge, and if we cross it positively, we increase the winding number of the star $S$ of $\Lambda$ containing $e$; if we cross $e$ negatively, we decrease the winding number of $S$.

(iii) If $e$ is $k$-relevant, then we decrease the winding number of one star of $\Lambda$ containing $e$ and increase the winding number of the other star of $\Lambda$ containing $e$.

Let us give a formal proof in the dual:

Proof of Proposition 32 Both $\sigma_{\lambda}(q)$ and $\delta^{k}(q)$ can be read on the pseudoline $q^{*}$ :

(i) If $\tau_{\lambda}(q)$ denotes the number of intersection points between $q^{*}$ and $\lambda$ (that is, the number of tangents to $S(\lambda)$ passing through $q)$, then $\sigma_{\lambda}(q)=\left(\tau_{\lambda}(q)-1\right) / 2$.

(ii) If $\gamma^{k}(q)$ denotes the number of intersection points between $q^{*}$ and the first $k$ levels of $P^{*}$, then $\delta^{k}(q)=k-\gamma^{k}(q) / 2$.

The pseudoline $q^{*}$ has exactly $|P|$ crossings with $P^{*}$ (since $P^{*} \cup\left\{q^{*}\right\}$ is an arrangement), which are crossings either with the pseudolines of $\Lambda$ or with the first $k$ levels of $P^{*}$. Hence,

$$
|P|=\gamma^{k}(q)+\sum_{\lambda \in \Lambda} \tau_{\lambda}(q)=2 k-2 \delta^{k}(q)+|\Lambda|+2 \sum_{\lambda \in \Lambda} \sigma_{\lambda}(q),
$$

and we get the aforementioned result since $|\Lambda|=|P|-2 k$.

Remark 33 As a consequence of Proposition 32, we see that the $k$-depth of any point $q$ in any point set $P$ is always non-negative (as a sum of non-negative numbers). It is interesting to notice that Welzl proved in [44] that this non-negativity property is actually equivalent to the Lower Bound Theorem for $d$-dimensional polytopes with $d+3$ vertices.

A corner of the star $S(\lambda)$ is an internal convex angle of it (see Fig. 21(a)). In the following proposition, we are interested in the number of corners of $S(\lambda)$. For points in convex position (Sect. 3.3), the number of corners of a star is always $2 k+1$. This is not true anymore in general position as illustrated in Fig. 20. The following proposition gives our best bounds on the number of corners of the star of a multipseudotriangulation.

Proposition 34 The number of corners of a star $S(\lambda)$ of a $k$-pseudotriangulation of $P^{*}$ is odd and between $2 k+1$ and $2(k-1)|P|+2 k+1$.

Proof We read convexity of internal angles of $S(\lambda)$ on the preimage $\bar{\lambda}$ of the pseudoline $\lambda$ under the projection $\pi$. Let $p q r$ be an internal angle, let $v=p^{*} \wedge q^{*}$ and $w=q^{*} \wedge r^{*}$ denote the contact points corresponding to the two edges $[p, q]$ and $[q, r]$ 

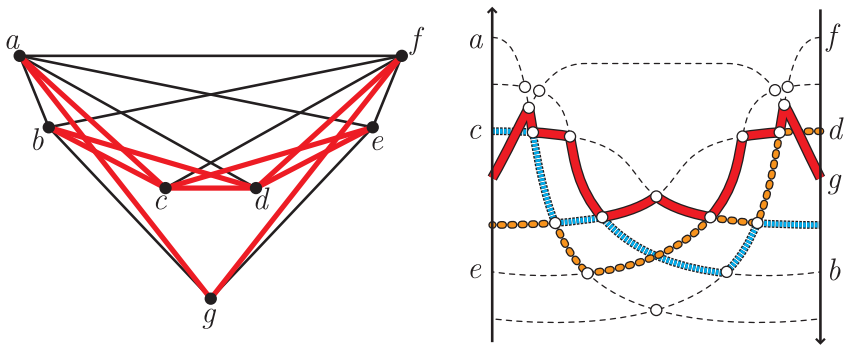

Fig. 20 A star of a 2-pseudotriangulation of seven points with nine corners. This example can be generalized to a 2-pseudotriangulation of $n$ points with $2 n-5$ corners

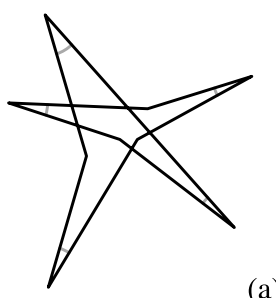

(a)

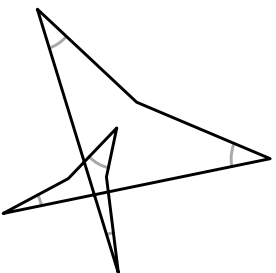

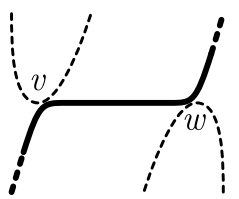

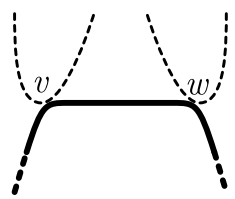

(b)

Fig. 21 (a) Two stars with five corners. (b) The two possible configurations of two consecutive contact points on $\lambda$ : convex (left) and concave (right)

of this angle, and let $\bar{v}$ and $\bar{w}$ denote two consecutive preimages of $v$ and $w$ on $\bar{\lambda}$ (meaning that $\bar{w}$ is located between $\bar{v}$ and $\tau(\bar{v})$ ). The angle pqr is a corner if and only if $\bar{v}$ and $\bar{w}$ lie on opposite sides of $\bar{\lambda}$, meaning that the other curves touching $\bar{\lambda}$ at $\bar{v}$ and $\bar{w}$ lie on opposite sides, one above and one below $\bar{\lambda}$ (see Fig. 21(b)).

In particular, the number $c(\lambda)=c$ of corners of $S(\lambda)$ is the number of opposite consecutive contact points on $\bar{\lambda}$ between two versions $\bar{v}$ and $\tau(\bar{v})$ of a contact point $v$ of $\lambda$. To see that $c$ is odd, imagine that we are discovering the contact points of $\lambda$ one by one. The first contact point $v$ that we see corresponds to two opposite contact points $\bar{v}$ and $\tau(\bar{v})$ on $\bar{\lambda}$. Then, at each stage, we insert a new contact point $\bar{w}$ between two old contact points that can be:

(i) either on opposite sides and then we are not changing $c$;

(ii) or on the same side and we are adding to $c$ either 0 (if $\bar{w}$ is also on the same side) or 2 (if $\bar{w}$ is on the opposite side).

Thus, $c$ remains odd in any case.

To prove the lower bound, we use our witness method. We perturb $\lambda$ a little bit to obtain a pseudoline $\mu$ that passes on the opposite side of each contact point (this is possible since $c$ is odd). This pseudoline $\mu$ crosses $\lambda$ between each pair of opposite contact points and crosses the other pseudolines of $\Lambda$ exactly as $\lambda$ does. Thus, $\mu$ crosses $\Lambda$ exactly $|\Lambda|-1+c$ times. But since $\mu$ is a pseudoline, it has to cross all the pseudolines of $P^{*}$ at least once. Thus, $|P| \leq|\Lambda|-1+c=|P|-2 k-1+c$ and $c \geq 2 k+1$. 
From this lower bound, we derive automatically the upper bound. Indeed, we know that the number of corners around one point $p$ is at $\operatorname{most} \operatorname{deg}(p)-1$. Consequently,

$$
\sum_{p \in P}(\operatorname{deg}(p)-1) \geq \sum_{\nu \in \Lambda} c(\nu)=c(\lambda)+\sum_{\substack{\nu \in \Lambda \\ \nu \neq \lambda}} c(\nu) .
$$

The left sum equals $2 k(2|P|-2 k-1)-|P|$ while, according to the previous lower bound, the right one is at least $c+(|P|-2 k-1)(2 k+1)$. Thus we get $c \leq 2(k-1)|P|+2 k+1$.

\section{Iterated Multipseudotriangulations}

By definition, a $k$-pseudotriangulation of an $m$-pseudotriangulation of a pseudoline arrangement $L$ is a $(k+m)$-pseudotriangulation of $L$. In this section, we study these iterated sequences of multipseudotriangulations. In particular, we compare multipseudotriangulations with iterated sequences of 1-pseudotriangulations.

\subsection{Definition and Examples}

Let $L$ be a pseudoline arrangement. An iterated multipseudotriangulation of $L$ is a sequence $\Lambda_{1}, \ldots, \Lambda_{r}$ of pseudoline arrangements such that $\Lambda_{i}$ is a multipseudotriangulation of $\Lambda_{i-1}$ for all $i$ (by convention, $\Lambda_{0}=L$ ). We call signature of $\Lambda_{1}, \ldots, \Lambda_{r}$ the sequence $k_{1}<\cdots<k_{r}$ of integers such that $\Lambda_{i}$ is a $k_{i}$-pseudotriangulation of $L$ for all $i$. Observe that the assumption that contact points of a pseudoline arrangement $L$ should be contact points of any multipseudotriangulation of $L$ is natural in this setting: iterated multipseudotriangulations correspond to decreasing sequences of sets of crossing points.

A decomposition of a multipseudotriangulation $\Lambda$ of a pseudoline arrangement $L$ is an iterated multipseudotriangulation $\Lambda_{1}, \ldots, \Lambda_{r}$ of $L$ such that $\Lambda_{r}=\Lambda$ and $r>1$. We say that $\Lambda$ is decomposable if such a decomposition exists, and irreducible otherwise. The decomposition is complete if its signature is $1,2, \ldots, r$.

It is tempting to believe that all multipseudotriangulations are completely decomposable. This would allow to focus only on pseudotriangulations. However, we start by showing that not even all multitriangulations are decomposable. The following example is due to Francisco Santos.

Example 35 (An irreducible 2-triangulation of the 15-gon) We consider the geometric graph $T$ of Fig. 22. The edges are:

(i) all the 2-irrelevant and 2-boundary edges of the 15-gon, and

(ii) the five zigzags $Z_{a}=\{[3 a, 3 a+6],[3 a+6,3 a+1],[3 a+1,3 a+5]$, $[3 a+5,3 a+2]\}$, for $a \in\{0,1,2,3,4\}$.

Thus, $T$ has 50 edges and is 3-crossing-free (since the only 2-relevant edges of $T$ that cross a zigzag $Z_{a}$ are edges of $Z_{a-1}$ and $Z_{a+1}$ ). Consequently, $T$ is a 2-triangulation of the 15-gon. 
Fig. 22 An irreducible

2-triangulation of the 15-gon: it contains no triangulation

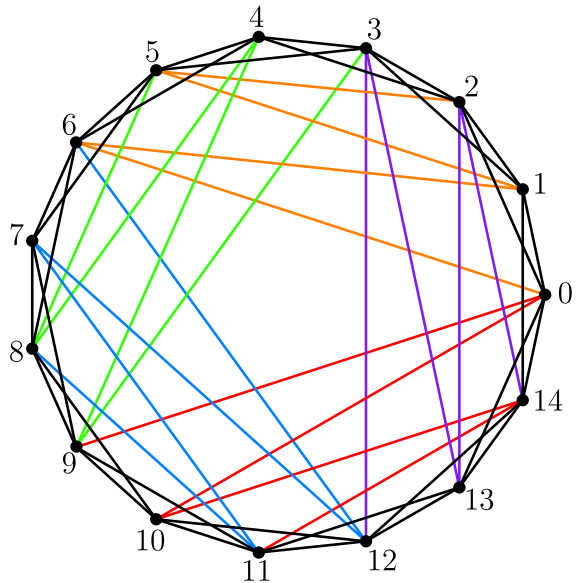

Let us now prove that $T^{*}$ is irreducible, that is, that $T$ contains no triangulation. Observe first that the edge $[0,6]$ cannot be an edge of a triangulation contained in $T$ since none of the triangles $06 i, i \in\{7, \ldots, 14\}$, is contained in $T$. Thus, we are looking for a triangulation contained in $T \backslash\{[0,6]\}$. Repeating the argument successively for the edges $[1,6],[1,5]$ and $[2,5]$, we prove that the zigzag $Z_{0}$ is disjoint from any triangulation contained in $T$. By symmetry, this proves the irreducibility of $T^{*}$.

\subsection{Iterated Greedy Pseudotriangulations}

Greedy multipseudotriangulations provide interesting examples of iteration of pseudotriangulations. Let $L$ be a pseudoline arrangement, and $\chi$ be a cut of $L$.

Theorem 36 For any positive integers $a$ and $b, \Gamma_{\chi}^{a+b}(L)=\Gamma_{\chi}^{b}\left(\Gamma_{\chi}^{a}(L)\right)$. Consequently, for any integer $k, \Gamma_{\chi}^{k}(L)=\Gamma_{\chi}^{1} \circ \Gamma_{\chi}^{1} \circ \cdots \circ \Gamma_{\chi}^{1}(L)$, where $\Gamma_{\chi}^{1}($.$) is iterated k$ times.

Proof Since $\chi$ is a cut of $L$, it is also a cut of $\Gamma_{\chi}^{a}(L)$ and thus $\Gamma_{\chi}^{b}\left(\Gamma_{\chi}^{a}(L)\right)$ is well defined. Observe also that we can assume that $L$ has no contact point (otherwise, we can open them). Let $n:=|L|$ and $m:=\left(\begin{array}{c}n \\ 2\end{array}\right)$.

Let $\chi=\chi_{0}, \ldots, \chi_{m}=\chi$ be a backward sweep of $L$. For all $i$, let $v_{i}$ denote the vertex of $L$ swept when passing from $\chi_{i}$ to $\chi_{i+1}$, and $i^{\square}$ denote the integer such that the pseudolines that cross at $v_{i}$ are the $i^{\square}$ th and $\left(i^{\square}+1\right)$ th pseudolines of $L$ on $\chi_{i}$.

Let $\sigma_{0}, \ldots, \sigma_{m}$ denote the sequence of permutations corresponding to $\Gamma_{\chi}^{a}(L)$ on the sweep $\chi_{0}, \ldots, \chi_{m}$. In other words, $\sigma_{0}$ is the permutation

$$
[1, \ldots, a, n-a, n-a-1, \ldots, a+2, a+1, n-a+1, \ldots, n],
$$

whose first $a$ and last $a$ entries are preserved, while its $n-2 a$ intermediate entries are inverted. Then, for all $i$, the permutation $\sigma_{i+1}$ is obtained from $\sigma_{i}$ by sorting its $i^{\square}$ th and $\left(i^{\square}+1\right)$ th entries.

Similarly, let $\rho_{0}, \ldots, \rho_{m}$ and $\omega_{0}, \ldots, \omega_{m}$ denote the sequences of permutations corresponding to $\Gamma_{\chi}^{a+b}(L)$ and $\Gamma_{\chi}^{b}\left(\Gamma_{\chi}^{a}(L)\right)$, respectively: both $\rho_{0}$ and $\omega_{0}$ equal the 
permutation whose first and last $a+b$ entries are preserved and whose $n-2 a-2 b$ intermediate entries are inverted, and:

- $\rho_{i+1}$ is obtained from $\rho_{i}$ by sorting its $i^{\square}$ th and $\left(i^{\square}+1\right)$ th entries;

- if $v_{i} \notin \Gamma_{\chi}^{a}(L)$, then $\omega_{i+1}$ is obtained from $\omega_{i}$ by sorting its $i^{\square}$ th and $\left(i^{\square}+1\right)$ th entries; otherwise, $\omega_{i+1}=\omega_{i}$.

We claim that for all $i$,

(A) all the inversions of $\rho_{i}$ are also inversions of $\sigma_{i}: \rho_{i}(p)>\rho_{i}(q)$ implies $\sigma_{i}(p)>\sigma_{i}(q)$ for all $1 \leq p<q \leq n$; and

(B) $\rho_{i}=\omega_{i}$.

We prove this claim by induction on $i$. It is clear for $i=0$. Assume it holds for $i$ and let us prove it for $i+1$. We have two possible situations:

(1) First case: $\sigma_{i}\left(i^{\square}\right)<\sigma_{i}\left(i^{\square}+1\right)$. Then $\sigma_{i+1}=\sigma_{i}$ and $v_{i} \in \Gamma_{\chi}^{a}(L)$. Thus, $\omega_{i+1}=\omega_{i}$. Furthermore, using property (A) at rank $i$, we know that $\rho_{i}\left(i^{\square}\right)<\rho_{i}\left(i^{\square}+1\right)$, and thus $\rho_{i+1}=\rho_{i}$. To summarize, $\sigma_{i+1}=\sigma_{i}, \omega_{i+1}=\omega_{i}$, and $\rho_{i+1}=\rho_{i}$, which trivially implies that properties (A) and (B) remain true.

(2) Second case: $\sigma_{i}\left(i^{\square}\right)>\sigma_{i}\left(i^{\square}+1\right)$. Then $\sigma_{i+1}$ is obtained from $\sigma_{i}$ by exchanging the $i^{\square}$ th and $(i \square+1)$ th entries, and $v_{i} \notin \Gamma_{\chi}^{a}(L)$. Consequently, $\rho_{i+1}$ and $\omega_{i+1}$ are both obtained from $\rho_{i}$ and $\omega_{i}$, respectively, by sorting their $i^{\square}$ th and $\left(i^{\square}+1\right)$ th entries. Thus, property (B) obviously remains true. As far as property (A) is concerned, the result is obvious if $p$ and $q$ are different from $i^{\square}$ and $i^{\square}+1$. By symmetry, it suffices to prove that for any $p<i^{\square}$, $\rho_{i+1}(p)>\rho_{i+1}\left(i^{\square}\right)$ implies $\sigma_{i+1}(p)>\sigma_{i+1}\left(i^{\square}\right)$. We have to consider two subcases:

(a) First subcase: $\rho_{i}\left(i^{\square}\right)<\rho_{i}\left(i^{\square}+1\right)$. Then $\rho_{i+1}=\rho_{i}$. Thus, if $p<i^{\square}$ is such that $\rho_{i+1}(p)>\rho_{i+1}\left(i^{\square}\right)$, then we have $\rho_{i}(p)>\rho_{i}\left(i^{\square}\right)$. Consequently, we obtain $\sigma_{i+1}(p)=\sigma_{i}(p)>\sigma_{i}\left(i^{\square}\right)>\sigma_{i}\left(i^{\square}+1\right)=\sigma_{i+1}\left(i^{\square}\right)$.

(b) Second subcase: $\rho_{i}\left(i^{\square}\right)>\rho_{i}\left(i^{\square}+1\right)$. Then $\rho_{i+1}$ is obtained from $\rho_{i}$ by exchanging its $i^{\square}$ th and $\left(i^{\square}+1\right)$ th entries. If $p<i^{\square}$ is such that $\rho_{i+1}(p)>\rho_{i+1}\left(i^{\square}\right)$, then we have $\rho_{i}(p)>\rho_{i}\left(i^{\square}+1\right)$. Consequently, we obtain $\sigma_{i+1}(p)=\sigma_{i}(p)>\sigma_{i}\left(i^{\square}+1\right)=\sigma_{i+1}\left(i^{\square}\right)$.

Obviously, property (B) of our claim proves the theorem.

\subsection{Flips in Iterated Multipseudotriangulations}

Let $\Lambda_{1}, \ldots, \Lambda_{r}$ be an iterated multipseudotriangulation of a pseudoline arrangement $L$, with signature $k_{1}<\cdots<k_{r}$. Let $v$ be a contact point of $\Lambda_{r}$ (which is not a contact point of $L$ ), and let $i$ denote the first integer for which $v$ is a contact point of $\Lambda_{i}$ (thus, $v$ is a contact point of $\Lambda_{j}$ if and only if $i \leq j \leq r$ ). For all $i \leq j \leq r$, let $\Lambda_{j}^{\prime}$ denote the pseudoline arrangement obtained from $\Lambda_{j}$ by flipping $v$, and let $w_{j}$ denote the new contact point of $\Lambda_{j}^{\prime}$. Let $j$ denote the biggest integer such that $w_{j}=w_{i}$. There are three possibilities:

(i) If $j=r$, then $\Lambda_{1}, \ldots, \Lambda_{i-1}, \Lambda_{i}^{\prime}, \ldots, \Lambda_{r}^{\prime}$ is an iterated multipseudotriangulation of $L$. We say that it is obtained from $\Lambda_{1}, \ldots, \Lambda_{r}$ by a complete flip of $v$. 


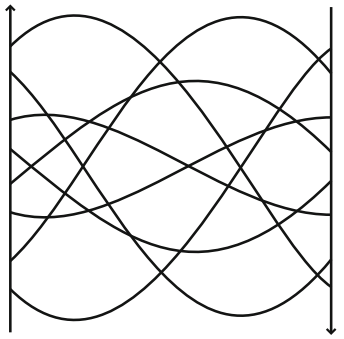

(a)

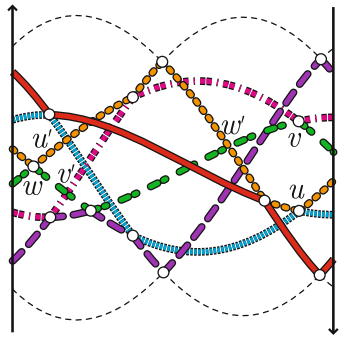

(b)

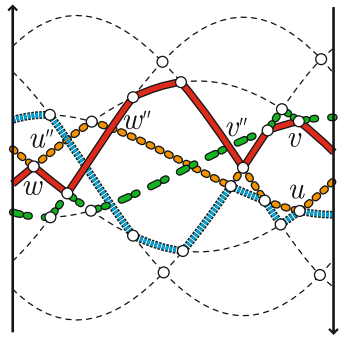

(c)

Fig. 23 An iterated multipseudotriangulation: a pseudoline arrangement $L$ (a), a 1-pseudotriangulation $\Lambda_{1}$ of $L(\mathbf{b})$, and a 1-pseudotriangulation $\Lambda_{2}$ of $\Lambda_{1}(\mathbf{c})$. The contact points $u, v, w$ illustrate the three possible situations for flipping a contact point

(ii) If $j<r$, and $w_{i}=w_{j}$ is a contact point of $\Lambda_{j+1}$, then

$$
\Lambda_{1}, \ldots, \Lambda_{i-1}, \Lambda_{i}^{\prime}, \ldots, \Lambda_{j}^{\prime}, \Lambda_{j+1}, \ldots, \Lambda_{r}
$$

is an iterated multipseudotriangulation of $L$. We say that it is obtained from $\Lambda_{1}, \ldots, \Lambda_{r}$ by a partial flip of $v$.

(iii) If $j<r$, and $w_{i}=w_{j}$ is a crossing point in $\Lambda_{j+1}$, then we cannot flip $v$ in $\Lambda_{i}$ maintaining an iterated multipseudotriangulation of $L$.

To illustrate these three possible cases, we have labeled on Fig. 23 some intersection points of an iterated pseudotriangulation. We have chosen three contact points $u, v, w$ in (b). For $z \in\{u, v, w\}$, we label $z^{\prime}$ (resp. $z^{\prime \prime}$ ) the crossing point corresponding to $z$ in (b) (resp. in (c)). Observe that:

(i) points $u^{\prime}$ and $u^{\prime \prime}$ coincide. Thus we can flip simultaneously point $u$ in (b) and (c) (complete flip);

(ii) points $v^{\prime}$ is different from $v^{\prime \prime}$ but is a contact point in (c). Thus, we can just flip $v$ in (b), without changing (c) and we preserve an iterated pseudotriangulation (partial flip);

(iii) point $w^{\prime}$ is a crossing point in (c), different from $w^{\prime \prime}$. Thus, we cannot flip $w$ in (b) maintaining an iterated pseudotriangulation.

Let $G^{k_{1}, \ldots, k_{r}}(L)$ be the graph whose vertices are the iterated multipseudotriangulations of $L$ with signature $k_{1}<\cdots<k_{r}$, and whose edges are the pairs of iterated multipseudotriangulations linked by a (complete or partial) flip.

Theorem 37 The graph of flips $G^{k_{1}, \ldots, k_{r}}(L)$ is connected.

To prove this proposition, we need the following lemma:

Lemma 38 Any intersection point $v$ in the k-kernel of a pseudoline arrangement is a contact point in a $k$-pseudotriangulation of it.

Proof The result holds when $k=1$. We obtain the general case by iteration. 
Proof of Theorem 37 We prove the result by induction on $r$ ( $L$ is fixed). When $r=1$, we already know that the flip graph is connected. Now, let $A_{-}$and $A_{+}$be two iterated multipseudotriangulations of $L$ with signature $k_{1}<\cdots<k_{r}$ that we want to join by flips. Let $B_{-}$and $B_{+}$be iterated multipseudotriangulations of $L$ with signature $k_{1}<$ $\cdots<k_{r-1}$, and $\Lambda_{-}$and $\Lambda_{+}$be $k_{r}$-pseudotriangulations of $L$ such that $A_{-}=B_{-}, \Lambda_{-}$ and $A_{+}=B_{+}, \Lambda_{+}$.

By induction, $G^{k_{1}, \ldots, k_{r-1}}(L)$ is connected: let

$$
B_{-}=B_{1}, B_{2}, \ldots, B_{p-1}, B_{p}=B_{+}
$$

be a path from $B_{-}$to $B_{+}$in $G^{k_{1}, \ldots, k_{r-1}}(L)$. For all $j$, let $v_{j}$ be such that $B_{j+1}$ is obtained from $B_{j}$ by flipping $v_{j}$ and let $w_{j}$ be such that $B_{j}$ is obtained from $B_{j+1}$ by flipping $w_{j}$. Let $\Lambda_{j}$ be a $k_{r}$-pseudotriangulation of $L$ containing the contact points of $B_{j}$ plus $w_{j}$ (it exists by Lemma 38), and let $C_{j}=B_{j}, \Lambda_{j}$. Let $D_{j}$ be the iterated multipseudotriangulation of $L$ obtained from the iterated pseudotriangulation $C_{j}$ by a partial flip of $v_{j}$. Finally, since $G^{k_{r}}\left(B_{j}\right)$ is connected, there is a path of complete flips from $D_{j-1}$ to $C_{j}$.

Merging all these paths, we obtain a global path from $A_{-}$to $A_{+}$: we transform $A_{-}$ into $C_{1}$ via a path of complete flips; then $C_{1}$ into $D_{1}$ by the partial flip of $v_{1}$; then $D_{1}$ into $C_{2}$ via a path of complete flips; then $C_{2}$ into $D_{2}$ by the partial flip of $v_{2}$; and so on.

\section{Further Topics}

We discuss here the extensions in the context of multipseudotriangulations of two known results on pseudotriangulations:

(1) The first one concerns the connection between the greedy pseudotriangulation of a point set and its horizon trees.

(2) The second one extends to arrangements of double pseudolines the definition and properties of multipseudotriangulations.

\subsection{Greedy Multipseudotriangulations and Horizon Graphs}

We have seen in previous sections that the greedy $k$-pseudotriangulation of a pseudoline arrangement $L$ can be seen as:

(1) the unique source of the graph of increasing flips;

(2) a greedy choice of crossing points given by a sorting network;

(3) a greedy choice of contact points;

(4) an iteration of greedy 1-pseudotriangulations.

In this section, we provide a "pattern avoiding" characterization of the crossing points of the greedy $k$-pseudotriangulation of $L$.

Let $L$ be a pseudoline arrangement, and $\chi$ be a cut of $L$. We index by $\ell_{1}, \ldots, \ell_{n}$ the pseudolines of $L$ in the order in which they cross $\chi$ (it is well defined, up to a complete inversion). 
Fig. 24 The sets $\mathbb{U}_{\chi}^{k}(L)$, $\mathbb{L}_{\chi}^{k}(L)$, and $\mathbb{G}_{\chi}^{k}(L)$ for the pseudoline arrangement of Fig. 7(b) and $k \in\{1,2\}$. The underlying

$k$-pseudotriangulation is the greedy $k$-pseudotriangulation of $L$
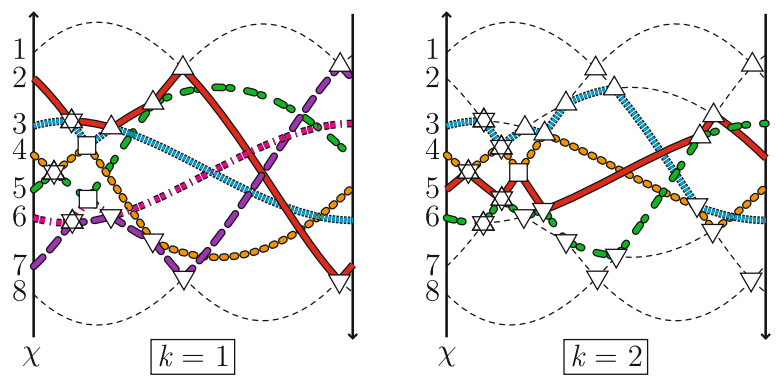

We define the $k$-upper $\chi$-horizon set of $L$ to be the set $\mathbb{U}_{\chi}^{k}(L)$ of crossing points $\ell_{\alpha} \wedge \ell_{\beta}$, with $1 \leq \alpha<\beta \leq n$, such that there is no $\gamma_{1}, \ldots, \gamma_{k}$ satisfying $\alpha<\gamma_{1}<\cdots<\gamma_{k}$ and $\ell_{\alpha} \wedge \ell_{\gamma_{i}} \preccurlyeq \chi \ell_{\alpha} \wedge \ell_{\beta}$ for all $i \in[k]$. In other words, on each pseudoline $\ell_{\alpha}$ of $L$, the set $\mathbb{U}_{\chi}^{k}(L)$ consists of the smallest $k$ crossing points of the form $\ell_{\alpha} \wedge \ell_{\beta}$, with $\alpha<\beta$.

Similarly, define the $k$-lower $\chi$-horizon set of $L$ to be the set $\mathbb{L}_{\chi}^{k}(L)$ of crossing points $\ell_{\alpha} \wedge \ell_{\beta}$, with $1 \leq \alpha<\beta \leq n$, such that there is no $\delta_{1}, \ldots, \delta_{k}$ satisfying $\delta_{1}<$ $\cdots<\delta_{k}<\beta$ and $\ell_{\beta} \wedge \ell_{\delta_{i}} \preccurlyeq \chi \ell_{\alpha} \wedge \ell_{\beta}$ for all $i \in[k]$. On each pseudoline $\ell_{\beta}$ of $L$, the set $\mathbb{L}_{\chi}^{k}(L)$ consists of the smallest $k$ crossing points of the form $\ell_{\alpha} \wedge \ell_{\beta}$, with $\alpha<\beta$.

Finally, we define the set $\mathbb{G}_{\chi}^{k}(L)$ to be the set of crossing points $\ell_{\alpha} \wedge \ell_{\beta}$, with $1 \leq \alpha<\beta \leq n$, such that there is no $\gamma_{1}, \ldots, \gamma_{k}$ and $\delta_{1}, \ldots, \delta_{k}$ satisfying:

(i) $\alpha<\gamma_{1}<\cdots<\gamma_{k}, \delta_{1}<\cdots<\delta_{k}<\beta$, and $\delta_{k}<\gamma_{1}$; and

(ii) $\ell_{\alpha} \wedge \ell_{\gamma_{i}} \preccurlyeq \chi \ell_{\alpha} \wedge \ell_{\beta}$ and $\ell_{\beta} \wedge \ell_{\delta_{i}} \preccurlyeq \chi \ell_{\alpha} \wedge \ell_{\beta}$ for all $i \in[k]$.

Obviously, the sets $\mathbb{U}_{\chi}^{k}(L)$ and $\mathbb{L}_{\chi}^{k}(L)$ are both contained in $\mathbb{G}_{\chi}^{k}(L)$.

Example 39 In Fig. 24, we have labeled the vertices of the pseudoline arrangement $L$ of Fig. 7(b) with different geometric tags according to their status:

$(\triangle)$ crossing points of the $k$-upper $\chi$-horizon set $\mathbb{U}_{\chi}^{k}(L)$ are represented by up triangles $\triangle$;

$(\nabla)$ crossing points of the $k$-lower $\chi$-horizon set $\mathbb{L}_{\chi}^{k}(L)$ are represented by down triangles $\nabla$;

$(\notin)$ crossing points in both $\mathbb{U}_{\chi}^{k}(L)$ and $\mathbb{L}_{\chi}^{k}(L)$ are represented by up and down triangles $\nLeftarrow$;

( $\square$ ) crossing points of $\mathbb{G}_{\chi}^{k}(L)$ but neither in $\mathbb{U}_{\chi}^{k}(L)$, nor in $\mathbb{L}_{\chi}^{k}(L)$ are represented by squares $\square$.

Observe that the remaining vertices are exactly the crossing points of the $\chi$-greedy $k$-pseudotriangulation of $L$.

Example 40 We consider the arrangement $C_{n}^{*}$ of $n$ pseudolines in convex position. Let $z$ be a vertex on the upper hull of its support, $F:=\left\{z^{\prime} \mid z \preccurlyeq z^{\prime}\right\}$ denote the filter generated by $z$, and $\chi$ denote the corresponding cut (see Fig. 25). It is easy to check that: 
Fig. 25 The sets $\mathbb{U}_{\chi}^{k}\left(C_{8}^{*}\right)$, $\mathbb{L}_{\chi}^{k}\left(C_{8}^{*}\right)$, and $\mathbb{G}_{\chi}^{k}\left(C_{8}^{*}\right)$ for the arrangement $C_{8}^{*}$ of eight pseudolines in convex position, and $k \in\{1,2\}$. The underlying $k$-pseudotriangulation is the greedy $k$-pseudotriangulation of $C_{8}^{*}$

Fig. 26 A mutation in the arrangement of Fig. 7(b)
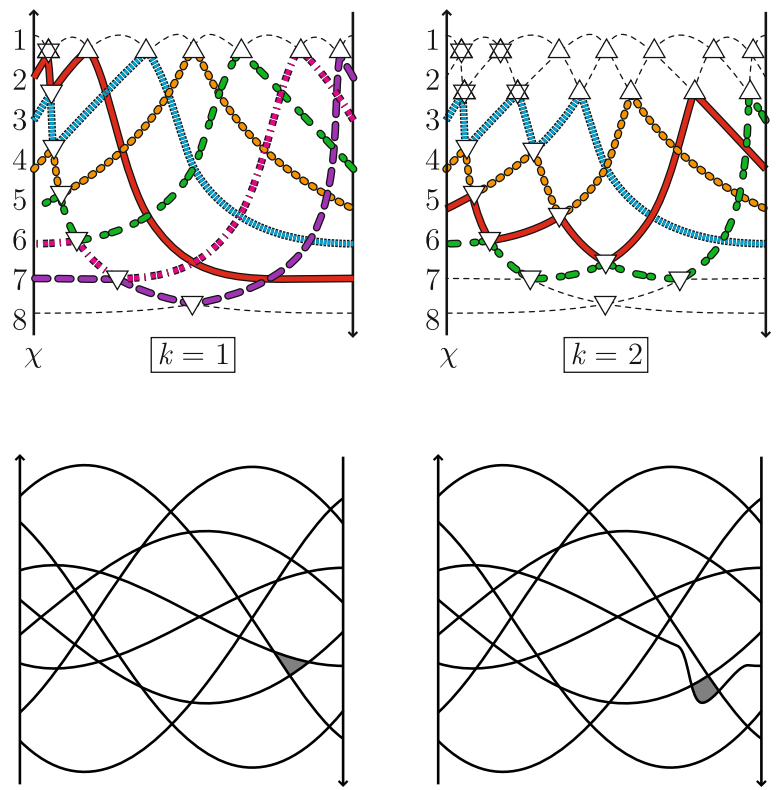

(i) $\mathbb{U}_{\chi}^{k}\left(C_{n}^{*}\right)=\left\{\ell_{\alpha} \wedge \ell_{\beta} \mid 1 \leq \alpha \leq n\right.$ and $\left.\alpha<\beta \leq \alpha+k\right\}$;

(ii) $\mathbb{L}_{\chi}^{k}\left(C_{n}^{*}\right)=\left\{\ell_{\alpha} \wedge \ell_{\beta} \mid 1 \leq \alpha \leq k\right.$ and $\left.\alpha<j \leq n\right\}$; and

(iii) $\mathbb{G}_{\chi}^{k}\left(C_{n}^{*}\right)=\mathbb{U}_{\chi}^{k}\left(\mathcal{C}_{n}\right) \cup \mathbb{L}_{\chi}^{k}\left(\mathcal{C}_{n}\right)$.

Observe again that the remaining vertices are exactly the crossing points of the $\chi$-greedy $k$-pseudotriangulation of $C_{n}^{*}$.

Theorem 41 extends this observation to all pseudoline arrangements, using convex position as a starting point for a proof by mutation.

Theorem 41 For any pseudoline arrangement $L$ with no contact point, and any cut $\chi$ of $L$, the sets $V\left(\Gamma_{\chi}^{k}(L)\right)$ and $\mathbb{G}_{\chi}^{k}(L)$ coincide.

The proof of this theorem works by mutation. A mutation is a local transformation of an arrangement $L$ that only inverts one triangular face of $L$. More precisely, it is a homotopy of arrangements during which only one curve $\ell \in L$ moves, sweeping a single vertex of the remaining arrangement $L \backslash\{\ell\}$ (see Fig. 26).

If $P$ is a point set of a topological plane, mutating an empty triangle $p^{*} q^{*} r^{*}$ of $P^{*}$ by sweeping the vertex $q^{*} \wedge r^{*}$ with the pseudoline $p^{*}$ corresponds in the primal to moving $p$ a little bit such that only the orientation of the triangle $p q r$ changes.

The graph of mutations on pseudoline arrangements is known to be connected: any two pseudoline arrangements (with no contact points and the same number of pseudolines) are homotopic via a finite sequence of mutations (followed by a homeomorphism). In fact, one can even avoid mutations of triangles that cross a given cut of $L$ : 
Fig. 27 A local image of a mutation
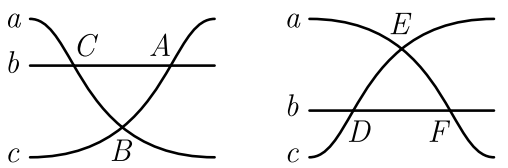

Proposition 42 Let $L$ and $L^{\prime}$ be two pseudoline arrangements of $\mathcal{M}$ (with no contact points and the same number of pseudolines) and $\chi$ be a cut of both of $L$ and $L^{\prime}$. There is a finite sequence of mutations of triangles disjoint from $\chi$ that transforms $L$ into $L^{\prime}$.

Proof We prove that any arrangement $L$ of $n$ pseudolines can be transformed into the arrangement $C_{n}^{*}$ of $n$ pseudolines in convex position (see Fig. 25).

Let $\ell_{1}, \ldots, \ell_{n}$ denote the pseudolines of $L$ (ordered by their crossings with $\chi$ ). Let $\Delta$ denote the triangle formed by $\chi, \ell_{1}$ and $\ell_{2}$. If there is a vertex of the arrangement $L \backslash\left\{\ell_{1}, \ell_{2}\right\}$ inside $\Delta$, then there is a triangle of the arrangement $L$ inside $\Delta$ and adjacent to $\ell_{1}$ or $\ell_{2}$. Mutating this triangle reduces the number of vertices of $L \backslash\left\{\ell_{1}, \ell_{2}\right\}$ inside $\Delta$ such that after some mutations, there is no more vertex inside $\Delta$. If $\Delta$ is intersected by pseudolines of $L \backslash\left\{\ell_{1}, \ell_{2}\right\}$, then there is a triangle inside $\Delta$ formed by $\ell_{1}, \ell_{2}$ and one of these intersecting pseudolines (the one closest to $\ell_{1} \wedge \ell_{2}$ ). Mutating this triangle reduces the number of pseudolines intersecting $\Delta$. Thus, after some mutations, $\Delta$ is a triangle of the arrangement $L$.

Repeating these arguments, we prove that for all $i \in\{2, \ldots, n-1\}$ and after some mutations, $\ell_{i}, \ell_{1}, \ell_{i+1}$ and $\chi$ delimit a face of the arrangement $L$. Thus, one of the two topological disk delimited by $\chi$ and $\ell_{1}$ contains no more vertex of $L$, and the proof is then straightforward by induction.

Let $\nabla$ be a triangle of $L$ not intersecting $\chi$. Let $L^{\prime}$ denote the pseudoline arrangement obtained from $L$ by mutating the triangle $\nabla$ into the inverted triangle $\Delta$. Let $a<b<c$ denote the indices of the pseudolines $\ell_{a}, \ell_{b}$ and $\ell_{c}$ that form $\nabla$ and $\Delta$. In $\nabla$, we denote $A=\ell_{b} \wedge \ell_{c}, B=\ell_{a} \wedge \ell_{c}$ and $C=\ell_{a} \wedge \ell_{b}$; similarly, in $\Delta$, we denote $D=\ell_{b} \wedge \ell_{c}, E=\ell_{a} \wedge \ell_{c}$ and $F=\ell_{a} \wedge \ell_{b}$ (see Fig. 27).

Lemma 43 With these notations, the following properties hold:

$$
\text { (i) } \begin{aligned}
B \in \mathbb{U}_{\chi}^{k}(L) & \Longleftrightarrow C \in \mathbb{U}_{\chi}^{k-1}(L) \Longleftrightarrow E \in \mathbb{U}_{\chi}^{k-1}\left(L^{\prime}\right) \\
& \Longleftrightarrow F \in \mathbb{U}_{\chi}^{k}\left(L^{\prime}\right), \\
A \in \mathbb{U}_{\chi}^{k}(L) & \Longleftrightarrow D \in \mathbb{U}_{\chi}^{k}\left(L^{\prime}\right), \\
E \in \mathbb{L}_{\chi}^{k}\left(L^{\prime}\right) & \Longleftrightarrow D \in \mathbb{L}_{\chi}^{k-1}\left(L^{\prime}\right) \Longleftrightarrow B \in \mathbb{L}_{\chi}^{k-1}(L) \\
& \Longleftrightarrow A \in \mathbb{L}_{\chi}^{k}(L), \\
F \in \mathbb{L}_{\chi}^{k}\left(L^{\prime}\right) & \Longleftrightarrow C \in \mathbb{L}_{\chi}^{k}(L) .
\end{aligned}
$$


(ii) $\quad C \in \mathbb{U}_{\chi}^{k}(L) \Longrightarrow A \in \mathbb{U}_{\chi}^{k}(L)$,

$E \in \mathbb{U}_{\chi}^{k}\left(L^{\prime}\right) \Longrightarrow D \in \mathbb{U}_{\chi}^{k}\left(L^{\prime}\right)$,

$D \in \mathbb{L}_{\chi}^{k}\left(L^{\prime}\right) \Longrightarrow F \in \mathbb{L}_{\chi}^{k}\left(L^{\prime}\right)$,

$B \in \mathbb{L}_{\chi}^{k}(L) \Longrightarrow C \in \mathbb{L}_{\chi}^{k}(L)$,

$B \in \mathbb{G}_{\chi}^{k}(L) \Longrightarrow C \in \mathbb{G}_{\chi}^{k}(L) \wedge D \in \mathbb{G}_{\chi}^{k}\left(L^{\prime}\right) \wedge F \in \mathbb{G}_{\chi}^{k}\left(L^{\prime}\right)$,

$E \in \mathbb{G}_{\chi}^{k}\left(L^{\prime}\right) \Longrightarrow D \in \mathbb{G}_{\chi}^{k}\left(L^{\prime}\right) \wedge C \in \mathbb{G}_{\chi}^{k}(L) \wedge A \in \mathbb{G}_{\chi}^{k}(L)$.

(iii)

$C \in \mathbb{G}_{\chi}^{k}(L) \Longrightarrow A \in \mathbb{U}_{\chi}^{k}(L) \vee C \in \mathbb{L}_{\chi}^{k}(L)$,

$D \in \mathbb{G}_{\chi}^{k}\left(L^{\prime}\right) \Longrightarrow F \in \mathbb{L}_{\chi}^{k}\left(L^{\prime}\right) \vee D \in \mathbb{U}_{\chi}^{k}\left(L^{\prime}\right)$,

$A \in \mathbb{G}_{\chi}^{k}(L) \Longrightarrow A \in \mathbb{U}_{\chi}^{k}(L) \vee C \in \mathbb{L}_{\chi}^{k-1}(L)$,

$F \in \mathbb{G}_{\chi}^{k}\left(L^{\prime}\right) \Longrightarrow F \in \mathbb{L}_{\chi}^{k}\left(L^{\prime}\right) \vee D \in \mathbb{U}_{\chi}^{k-1}\left(L^{\prime}\right)$.

(iv)
$C \in \mathbb{G}_{\chi}^{k}(L) \wedge E \notin \mathbb{G}_{\chi}^{k}\left(L^{\prime}\right) \Longrightarrow A \notin \mathbb{G}_{\chi}^{k}(L)$,
$D \in \mathbb{G}_{\chi}^{k}\left(L^{\prime}\right) \wedge B \notin \mathbb{G}_{\chi}^{k}(L) \Longrightarrow F \notin \mathbb{G}_{\chi}^{k}\left(L^{\prime}\right)$.

Proof By symmetry, it is enough to prove the first line of each of the four points of the lemma.

Properties of point (i) directly come from the definitions. For example, all the assertions of the first line are false if and only if there exist $\gamma_{1}, \ldots, \gamma_{k-1}$ with $a<$ $\gamma_{1}<\cdots<\gamma_{k-1}$ and, for all $i \in[k-1], \ell_{a} \wedge \ell_{\gamma_{i}} \preccurlyeq \chi C$ (or equivalently $\ell_{a} \wedge \ell_{\gamma_{i}} \preccurlyeq \chi E$ ).

We derive point (ii) from the following observation: if $\gamma>b$ and if $\ell_{b} \wedge \ell_{\gamma} \preccurlyeq{ }_{\chi} C$, then $\gamma>a$ and $\ell_{a} \wedge \ell_{\gamma} \preccurlyeq{ }_{\chi} B$.

For point (iii), assume that $A \notin \mathbb{U}_{\chi}^{k}(L)$ and $C \notin \mathbb{L}_{\chi}^{k}(L)$. Then there exist $\gamma_{1}, \ldots, \gamma_{k}$ and $\delta_{1}, \ldots, \delta_{k}$ such that $\delta_{1}<\cdots<\delta_{k}<b<\gamma_{1}<\cdots<\gamma_{k}$ and, for all $i \in[k], \ell_{b} \wedge$ $\ell_{\gamma_{i}} \preccurlyeq \chi A$ (and therefore $\ell_{a} \wedge \ell_{\gamma_{i}} \preccurlyeq \chi C$ ) and $\ell_{b} \wedge \ell_{\delta_{i}} \preccurlyeq \chi C$. Thus $C \notin \mathbb{G}_{\chi}^{k}(L)$.

Finally, assume that $C \in \mathbb{G}_{\chi}^{k}(L)$ and $E \notin \mathbb{G}_{\chi}^{k}\left(L^{\prime}\right)$. Then there exist $\gamma_{1}, \ldots, \gamma_{k}$ and $\delta_{1}, \ldots, \delta_{k}$ such that $a<\gamma_{1}<\cdots<\gamma_{k}, \delta_{1}<\cdots<\delta_{k}<c, \delta_{k}<\gamma_{1}$, and for all $i \in$ $[k], \ell_{a} \wedge \ell_{\gamma_{i}} \preccurlyeq \chi E$ and $\ell_{c} \wedge \ell_{\delta_{i}} \preccurlyeq \chi E$. Since $C \in \mathbb{G}_{\chi}^{k}(L)$, we have $\delta_{k}>b$. Thus $b<\gamma_{1}<\cdots<\gamma_{k}$ and for all $i \in[k], \ell_{b} \wedge \ell_{\gamma_{i}} \preccurlyeq \chi A$ and $\ell_{c} \wedge \ell_{\delta_{i}} \preccurlyeq \chi A$. This implies that $A \notin \mathbb{G}_{C}^{k}(L)$.

We are now ready to establish the proof of Theorem 41:

Proof of Theorem 41 The proof works by mutation. We already observed the result when the pseudoline arrangement is in convex position (see Example 40 and Fig. 25). Proposition 42 ensures that any pseudoline arrangement can be reached from this convex configuration by mutations of triangles not intersecting $\chi$. Thus, it is sufficient to prove that such a mutation preserves the property.

Assume that $L$ is a pseudoline arrangement and $\chi$ is a cut of $L$, for which the result holds. Let $\nabla$ be a triangle of $L$ not intersecting $\chi$. Let $L^{\prime}$ denote the pseudoline arrangement obtained from $L$ by mutating the triangle $\nabla$ into the inverted triangle $\Delta$. Let $A, B, C$ and $D, E, F$ denote the vertices of $\nabla$ and $\Delta$ as indicated in Fig. 27. 

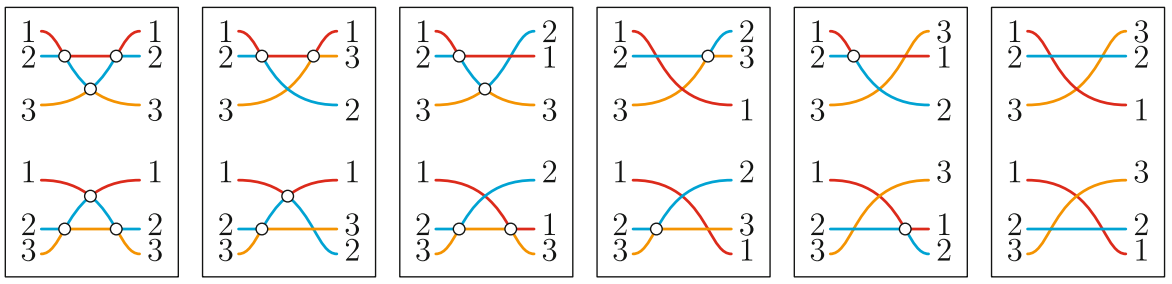

Fig. 28 The six possible cases for the mutation of the greedy $k$-pseudotriangulation

If $v$ is a vertex of the arrangement $L^{\prime}$ different from $D, E, F$, then:

$v \in V\left(\Gamma_{\chi}^{k}\left(L^{\prime}\right)\right) \Longleftrightarrow v \in V\left(\Gamma_{\chi}^{k}(L)\right) \Longleftrightarrow v \in \mathbb{G}_{\chi}^{k}(L) \Longleftrightarrow v \in \mathbb{G}_{\chi}^{k}\left(L^{\prime}\right)$.

Thus, we only have to prove the equivalence when $v \in\{D, E, F\}$. The proof is a (computational) case analysis: using the properties of Lemma 43 as boolean equalities relating the boolean variables defined by " $X \in \mathbb{Y}_{\chi}^{p}(L)$ " (where $X \in$ $\{A, B, C, D, E, F\}, \mathbb{Y} \in\{\mathbb{U}, \mathbb{L}, \mathbb{G}\}$, and $p \in\{k-1, k\})$, we have written a short boolean satisfiability program which affirms that:

(i) either $\{A, B, C\} \subset \mathbb{G}_{\chi}^{k}(L)$ and $\{D, E, F\} \subset \mathbb{G}_{\chi}^{k}\left(L^{\prime}\right)$;

(ii) or $\{A, B, C\} \cap \mathbb{G}_{\chi}^{k}(L)=\{A, C\}$ and $\{D, E, F\} \cap \mathbb{G}_{\chi}^{k}\left(L^{\prime}\right)=\{D, E\}$;

(iii) or $\{A, B, C\} \cap \mathbb{G}_{\chi}^{k}(L)=\{B, C\}$ and $\{D, E, F\} \cap \mathbb{G}_{\chi}^{k}\left(L^{\prime}\right)=\{D, F\}$;

(iv) or $\{A, B, C\} \cap \mathbb{G}_{\chi}^{k}(L)=\{A\}$ and $\{D, E, F\} \cap \mathbb{G}_{\chi}^{k}\left(L^{\prime}\right)=\{D\}$;

(v) or $\{A, B, C\} \cap \mathbb{G}_{\chi}^{k}(L)=\{C\}$ and $\{D, E, F\} \cap \mathbb{G}_{\chi}^{k}\left(L^{\prime}\right)=\{F\}$;

(vi) or $\{A, B, C\} \cap \mathbb{G}_{\chi}^{k}(L)=\emptyset$ and $\{D, E, F\} \cap \mathbb{G}_{\chi}^{k}\left(L^{\prime}\right)=\emptyset$.

It is easy to check that these six cases correspond to sorting the six possible permutations of $\{1,2,3\}$ on $\nabla$ and $\Delta$ (see Fig. 28). Consequently, if $V\left(\Gamma_{\chi}^{k}(L)\right) \cap$ $\{A, B, C\}=\mathbb{G}_{\chi}^{k}(L) \cap\{A, B, C\}$, then we have $V\left(\Gamma_{\chi}^{k}\left(L^{\prime}\right)\right) \cap\{D, E, F\}=\mathbb{G}_{\chi}^{k}\left(L^{\prime}\right) \cap$ $\{D, E, F\}$, which finishes the proof.

Let us finish this discussion by recalling the interpretation of the horizon sets when $k=1$. Let $P$ be a finite point set. For any $p \in P$, let $u(p)$ denote the point $q$ that minimizes the angle $(O x, p q)$ among all points of $P$ with $y_{p}<y_{q}$ (by convention, for the higher point $p$ of $P, u(p)=p)$. The upper horizon tree of $P$ is the set $\mathbb{U}(P)=\{p u(p) \mid p \in P\}$. The lower horizon tree $\mathbb{L}(P)$ of $P$ is defined symmetrically. See Fig. 29.

Choosing a cut $\chi$ of $P^{*}$ corresponding to the point at infinity $(-\infty, 0)$ makes coincide primal and dual definitions of horizon sets: we have $\mathbb{U}_{\chi}^{1}\left(P^{*}\right)=\mathbb{U}(P)^{*}$ and $\mathbb{L}_{\chi}^{1}\left(P^{*}\right)=\mathbb{L}(P)^{*}$.

In [31], Pocchiola observed that the set $\mathbb{U}(P) \cup \mathbb{L}(P)$ of edges can be completed into a pseudotriangulation of $P$ just by adding the sources of the faces of $P^{*}$ intersected by the cut $\chi$. The obtained pseudotriangulation is our $\chi$-greedy 1-pseudotriangulation $\Gamma_{\chi}^{1}\left(P^{*}\right)$. 

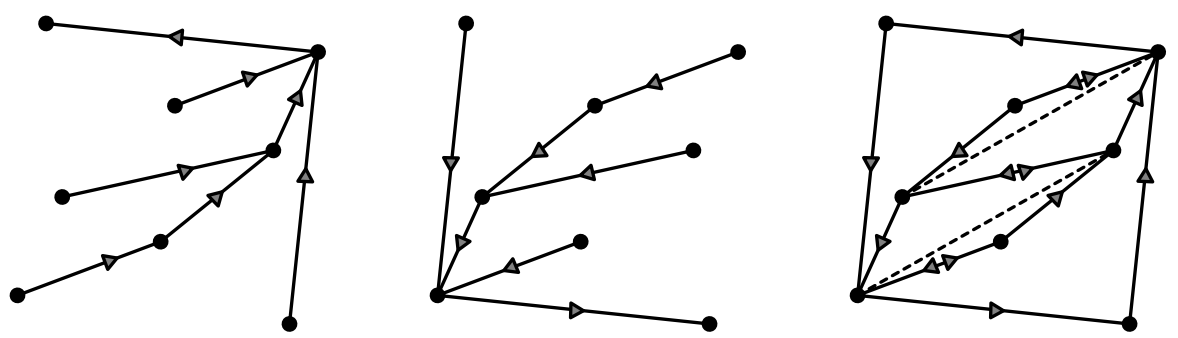

Fig. 29 The upper horizon tree (left), the lower horizon tree (middle), and the greedy pseudotriangulation (right) of the point set of Fig. 7(a). The dashed edges in the greedy pseudotriangulation are not in the horizon trees

Fig. 30 (a) A double pseudoline. (b) An arrangement of 2 double pseudolines

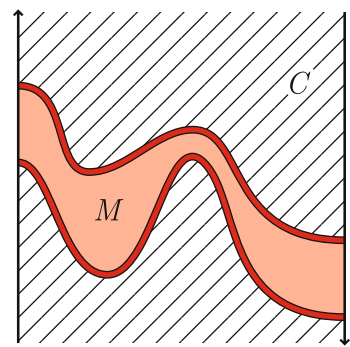

(a)

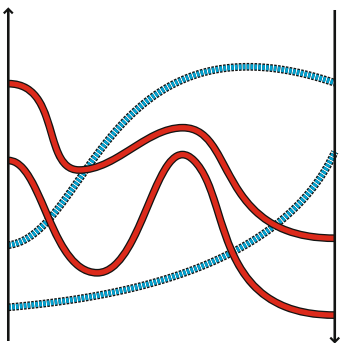

(b)

\subsection{Multipseudotriangulations of Double Pseudoline Arrangements}

In this section, we deal with double pseudoline arrangements, i.e. duals of sets of disjoint convex bodies. Definitions and properties of multipseudotriangulations naturally extend to these objects.

\subsubsection{Definitions}

A simple closed curve in the Möbius strip can be:

(i) either contractible (homotopic to a point);

(ii) or non separating, or equivalently homotopic to a generator of the fundamental group of $\mathcal{M}$ : it is a pseudoline;

(iii) or separating and non-contractible, or equivalently homotopic to the double of a generator of the fundamental group of $\mathcal{M}$ : it is called a double pseudoline (see Fig. 30(a)).

The complement of a double pseudoline $\ell$ has two connected components: the bounded one is a Möbius strip $M_{\ell}$ and the unbounded one is an open cylinder $C_{\ell}$ (see Fig. 30(a)).

The canonical example of a double pseudoline is the set $C^{*}$ of tangents to a convex body $C$ of the plane. Observe also that the $p$ th level of a pseudoline arrangement is a double pseudoline. If $C$ is a convex body of the plane, then the Möbius strip $M_{C^{*}}$ 
Fig. 31 A configuration of four disjoint convex bodies and its dual double pseudoline arrangement
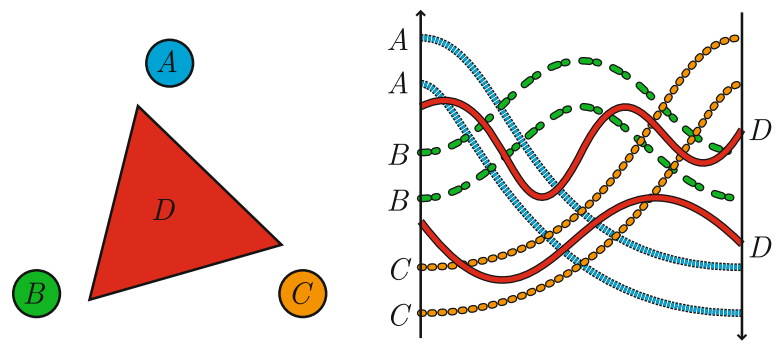

Fig. 32 A configuration of three disjoint convex bodies and its dual double pseudoline arrangement

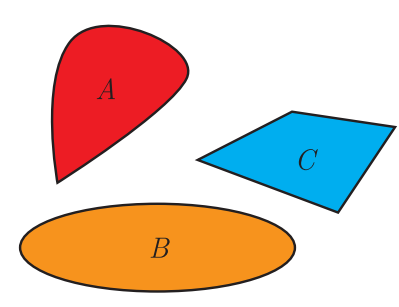

corresponds to lines that pierce $C$, while $C_{C^{*}}$ corresponds to lines disjoint from $C$. If $C$ and $C^{\prime}$ are two disjoint convex bodies, the two corresponding double pseudolines $C^{*}$ and $C^{*}$ cross four times (see Figs. 31 and 32). Each of these four crossings corresponds to one of the four bitangents (or common tangents) between $C$ and $C^{\prime}$.

Definition 44 [17] A double pseudoline arrangement is a finite set of double pseudolines such that any two of them have exactly four intersection points, cross transversally at these points, and induce a cell decomposition of the Möbius strip (i.e. the complement of their union is a union of topological disks, together with the external cell).

Given a set $Q$ of disjoint convex bodies in the plane (or in any topological plane), its dual $Q^{*}:=\left\{C^{*} \mid C \in Q\right\}$ is an arrangement of double pseudolines (see Figs. 31 and 32). Furthermore, as for pseudoline arrangements, any double pseudoline arrangement can be represented by (i.e. is the dual of) a set of disjoint convex bodies in a topological plane [17].

In this paper, we only consider simple arrangements of double pseudolines. Defining the support, the levels, and the kernels of double pseudoline arrangements as for pseudoline arrangements, we can extend multipseudotriangulations to double pseudoline arrangements (see Fig. 33):

Definition 45 A $k$-pseudotriangulation of a double pseudoline arrangement $L$ is a pseudoline arrangement supported by the $k$-kernel of $L$.

All the properties related to flips developed in Sect. 2 apply in this context. In the end of this section, we only revisit the properties of the primal of a multipseudotriangulation of a double pseudoline arrangement. 
Fig. 33 A pseudotriangulation of the set of disjoint convex bodies of Fig. 32
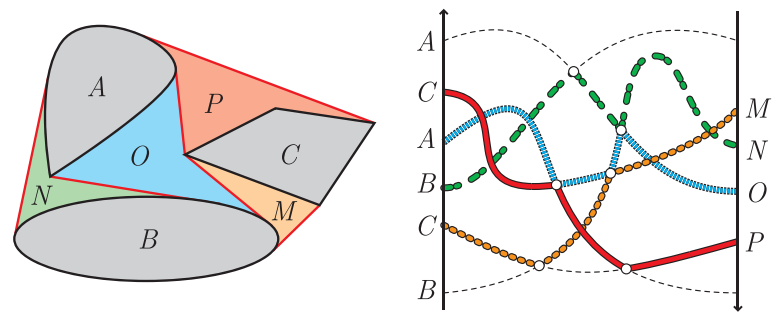

\subsubsection{Elementary Properties}

Let $Q$ be a set disjoint convex bodies in general position in the plane and $Q^{*}$ be its dual arrangement. Let $\Lambda$ be a $k$-pseudotriangulation of $Q^{*}, V(\Lambda)$ denote all crossing points of $Q^{*}$ that are not crossing points of $\Lambda$, and $E$ denote the corresponding set of bitangents of $Q$. As in Sect. 4.2, we discuss the properties of the primal configuration $E$ :

Lemma 46 The set $E$ has $4|Q| k-|Q|-2 k^{2}-k$ edges.

Proof The number of edges of $E$ is the number of crossing points of $Q^{*}$ minus the number of crossing points of $\Lambda$, i.e.

$$
\begin{aligned}
|E| & =4\left(\begin{array}{c}
\left|Q^{*}\right| \\
2
\end{array}\right)-\left(\begin{array}{c}
|\Lambda| \\
2
\end{array}\right)=4\left(\begin{array}{c}
|Q| \\
2
\end{array}\right)-\left(\begin{array}{c}
2|Q|-2 k \\
2
\end{array}\right) \\
& =4|Q| k-|Q|-2 k^{2}-k
\end{aligned}
$$

We now discuss pointedness. For any convex body $C$ of $Q$, we arbitrarily choose a point $p_{C}$ in the interior of $C$, and we consider the set $X_{C}$ of all segments between $p_{C}$ and a sharp boundary point of $C$. We denote by $X:=\bigcup_{C \in Q} X_{C}$ the set of all these segments.

\section{Lemma 47 The set $E \cup X$ cannot contain a k-alternation.}

Proof Let $C$ be a convex body of $Q$, let $q$ be a sharp point of $C$ and let $F:=\left\{\left[p_{i}, q\right] \mid\right.$ $i \in[2 k]\}$ be a set of edges incident to $q$ such that $\left\{\left[p_{C}, q\right]\right\} \cup F$ is a $k$-alternation. We prove that $F$ is not contained in $E$. Indeed, the dual pseudolines $\left\{p_{i}^{*} \mid i \in[2 k]\right\}$ intersect alternately the double pseudoline $C^{*}$ between the tangents to $C$ at $q$ (see Fig. 34). This ensures the existence of a witness pseudoline $\ell$ that separates all the contact points $p_{i}^{*} \wedge C^{*}$, while crossing $C^{*}$ exactly $2 k$ times and the other double pseudolines of $Q^{*}$ exactly has $q^{*}$ does. (As usual, we obtain it by a perturbation of the pseudoline $q^{*}$.) Counting the crossings of $\ell$ with $Q^{*}$ and $\Lambda$, respectively, we obtain:

(i) $\ell$ crosses $Q^{*}$ exactly $2\left|(Q \backslash\{C\})^{*}\right|+2 k=2|Q|+2 k-2$ times;

(ii) $\ell$ crosses $\Lambda$ at least $|\Lambda|=2|Q|-2 k$ times;

(iii) for each of the points $p_{i} \wedge q^{*}$, replacing the crossing point by a contact point removes two crossings with $\ell$. 

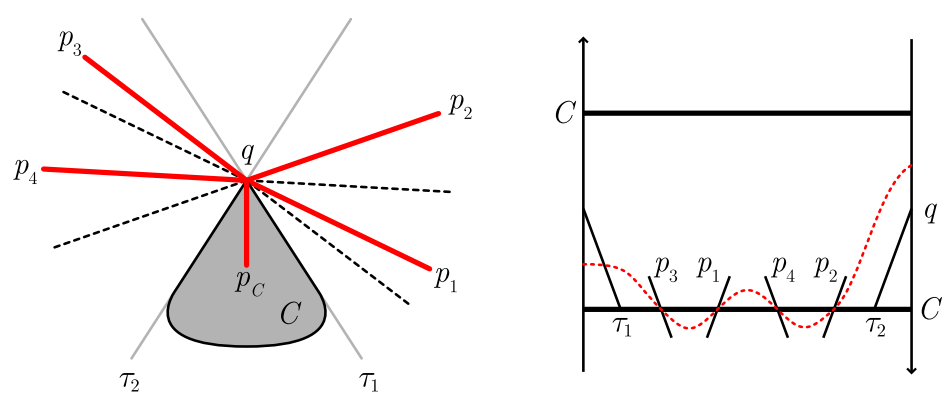

Fig. 34 A $k$-alternation at a sharp point

\subsubsection{Stars}

If $\lambda$ is a pseudoline of $\Lambda$, we call star the envelope $S(\lambda)$ of the primal lines of the points of $\lambda$. The star $S(\lambda)$ contains:

(i) all bitangents $\tau$ between two convex bodies of $Q$ such that $\tau$ is a contact point of $\lambda$; and

(ii) all convex arcs formed by the tangent points of the lines covered by $\lambda$ with the convex bodies of $Q$.

This star is a (non-necessarily simple) closed curve. We again have bounds on the number of corners (i.e. convex internal angles) of $S(\lambda)$ :

Proposition 48 The number of corners of $S(\lambda)$ is odd and between $2 k-1$ and $4 k|Q|-2 k-1$.

Proof In the case of double pseudoline arrangements, corners are even easier to characterize: a bitangent $\tau$ between two convex $C$ and $C^{\prime}$ of $Q$ always defines two corners, one at each extremity. These corners are contained in one of the two stars adjacent to $\tau$. Let $\lambda$ be a pseudoline with a contact point at $\tau$. In a neighborhood of $\tau$, the pseudoline $\lambda$ can be contained either in $M_{C^{*}}$ or in $C_{C^{*}}$. In the first case, the star $S(\lambda)$ contains the corner formed by the bitangent $\tau$ and the convex $C$ (or possibly, by the bitangent $\tau$ and another tangent to $C$ ); while in the second case, it does not. (The same observation holds for $C^{\prime}$.)

In other words, if the double pseudoline $C^{*}$ supports a pseudoline $\lambda$ between two contact points $v$ and $w$, then one of the three following situations occurs (see Fig. 35):

(i) either $v$ and $w$ lie on opposite sides of $\lambda$; then exactly one of these contact points lies in $M_{C^{*}}$, and $S(\lambda)$ has one corner at $C$,

(ii) or $v$ and $w$ both lie on $M_{C^{*}}$, and $S(\lambda)$ has two corners at $C$,

(iii) or $v$ and $w$ both lie on $C_{C^{*}}$, and $S(\lambda)$ has no corners at $C$.

In particular, the number $c=c(\lambda)$ of corners of $\lambda$ is the number of situations (i) plus twice the number of situations (ii). This proves that $c$ is odd and (at least) bigger than the number of opposite consecutive contact points of the pseudoline $\lambda$. 


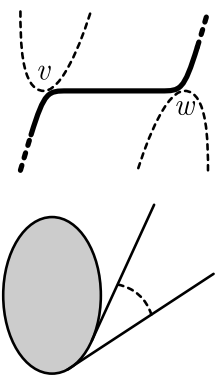

(i)
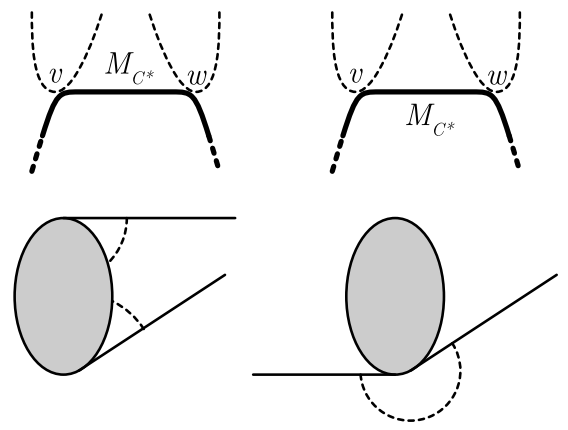

(iii)

Fig. 35 The three possible situations for two consecutive contact points on $\ell$

Fig. 36 A

2-pseudotriangulation of the double pseudoline arrangement of Fig. 31. Observe that the bolded red star has only 3 corners
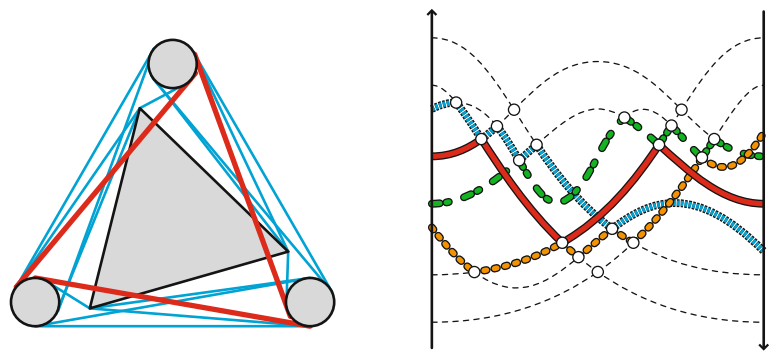

In order to get a lower bound on this number, we construct (as in the proof of Proposition 34) a witness pseudoline $\mu$ that crosses $\lambda$ between each pair of opposite contact points and passes on the opposite side of each contact point. It crosses $\lambda$ at most $c$ times and $\Lambda \backslash\{\lambda\}$ exactly $|\Lambda|-1$ times. Moreover, if $\alpha$ is a pseudoline and $\beta$ is a double pseudoline of $\mathcal{M}$, then either $\alpha$ is contained in $M_{\beta}$ and has no crossing with $\beta$, or $\alpha$ and $\beta$ have an even number of crossings. Since $\mu$ is a pseudoline and can be contained in at most one Möbius strip $M_{C}^{*}$ (for $C \in Q$ ), the number of crossings of $\mu$ with $Q^{*}$ is at least $2(|Q|-1)$. Thus, we obtain the lower bound $2(|Q|-1) \leq 2|Q|-2 k-1+c$, i.e. $c \geq 2 k-1$.

From this lower bound, we obtain the upper bound: the total number of corners is at most twice the number of bitangents:

$$
2\left(4 k|Q|-|Q|-2 k^{2}-k\right) \geq \sum_{\mu \in \Lambda} c(\mu) \geq c(\lambda)+(2|Q|-2 k-1)(2 k-1),
$$

and we get $c \leq 4|Q| k-2 k-1$.

When $k=1$, we can even prove that all stars are pseudotriangles. Indeed, since any star has at least three corners, the upper bound calculus gives $2(3|Q|-3) \geq$ $c+3(2|Q|-3)$, i.e. $c \leq 3$.

For general $k$, observe that contrary to the case of pseudoline arrangements, a star may have $2 k-1$ corners (see Fig. 36). 
Let us now give an analogue of Proposition 32. For any point $q$ in the plane, we denote by $\eta^{k}(q)$ the number of crossings between $q^{*}$ and the support of $Q^{*}$ minus its first $k$ levels. Let $\delta^{k}(q):=\eta^{k}(q) / 2-|Q|+k$. For any $\lambda \in \Lambda(U)$ and any point $q$ in the plane, we still denote by $\sigma_{\lambda}(q)$ the winding number of $S(\lambda)$ around $q$.

Proposition 49 For any point $q$ of the plane $\delta^{k}(q)=\sum_{\lambda \in \Lambda} \sigma_{\lambda}(q)$.

Proof Remember that if $\tau_{\lambda}(q)$ denotes the number of intersection points between $q^{*}$ and $\lambda$, then $\sigma_{\lambda}(q)=\left(\tau_{\lambda}(q)-1\right) / 2$. Thus, we have

$$
\eta^{k}(q)=\sum_{\lambda \in \Lambda} \tau_{\lambda}(q)=|\Lambda|+2 \sum_{\lambda \in \Lambda} \sigma_{\lambda}(q)
$$

and we get the result since $|\Lambda|=2|Q|-2 k$.

When $k=1$, it is easy to see that $\delta^{1}(q)$ is 1 if $q$ is inside the free space of the convex hull of $Q$ (i.e. in the convex hull of $Q$, but not in $Q$ ), and 0 otherwise. Remember that a pseudotriangulation of $Q$ is a pointed set of bitangents that decomposes the free space of the convex hull of $Q$ into pseudotriangles [33]. Propositions 48 and 49 provide, when $k=1$, the following analogue of Theorem 16:

Theorem 50 Let $Q$ be a set of disjoint convex bodies (in general position) and $Q^{*}$ denote its dual arrangement. Then:

(i) The dual arrangement $T^{*}:=\left\{\Delta^{*} \mid \Delta\right.$ pseudotriangle of $\left.T\right\}$ of a pseudotriangulation $T$ of $Q$ is a 1-pseudotriangulation of $Q^{*}$.

(ii) The primal set of edges

$$
E:=\left\{[p, q] \mid p, q \in P, p^{*} \wedge q^{*} \text { is not a crossing point of } \Lambda\right\}
$$

of a 1-pseudotriangulation $\Lambda$ of $Q^{*}$ is a pseudotriangulation of $Q$.

Observe that at least two other arguments are possible to prove (ii):

(1) either comparing the degrees of the flip graphs as in our first proof of Theorem 16;

(2) or checking that all forbidden configurations of the primal (two crossing bitangents, a non-pointed sharp vertex, a non-free bitangent) may not appear in the dual, as in our second proof of Theorem 16.

Let us finish this discussion about stars by interpreting the number $\delta^{k}(q)$ for general $k$ and for "almost every" point $q$. For any convex body $C$ of $Q$, let $\nabla C$ denote the intersection of all closed half-planes delimited by a bitangent between two convex bodies of $Q$, and containing $C$ (see Fig. 37). By definition, the bitangents between two convex bodies $C$ and $C^{\prime}$ of $Q$ coincide with the bitangent of $\nabla C$ and $\nabla C^{\prime}$. Furthermore, the convex bodies $\nabla C(C \in Q)$ are maximal for this property. We denote $\nabla Q:=\{\nabla C \mid C \in Q\}$ the set of maximal convex bodies of $Q$.

For a point $q$ outside $\nabla Q$, the interpretation of $\delta^{k}(q)$ is similar to the case of points. We call level of a bitangent $\tau$ the level of the corresponding crossing point in $Q^{*}$. Given a point $q$ outside $\nabla Q$, the number $\delta^{k}(q)$ is the number of bitangents 

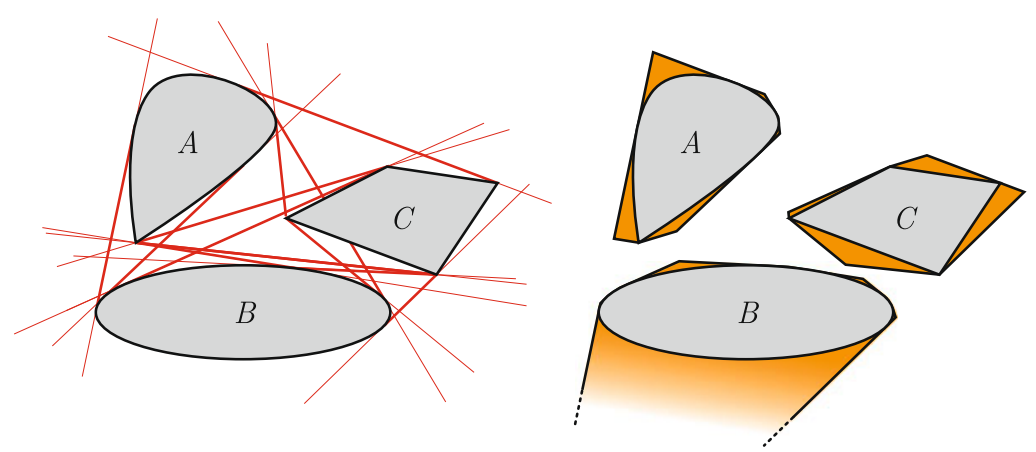

Fig. 37 The set of all bitangents to the arrangement of convex bodies of Fig. 32 and the corresponding maximal convex bodies

of level $k$ crossed by any (generic continuous) path from $q$ to the external face (in the complement of $\nabla Q$ ), counted positively when passing from the "big" side to the "small side", and negatively otherwise.

\section{Open Questions}

We finish by a short presentation of some open questions that have arisen out of this work. Since the submission of this paper, some of these questions were (at least partially) answered in [29, 40, 43]. We have decided to keep these questions in the discussion and to refer to the relevant articles in side remarks.

Primal of a Multipseudotriangulation When $k=1$ or in the case of convex position, primals of $k$-pseudotriangulations are characterized by simple non-crossing and pointedness conditions. For general $k$ and general position, we know that the primal of a $k$-pseudotriangulation is $k$-alternation-free (Lemma 27), but there exist $k$-pseudotriangulations containing $(k+1)$-crossings as well as $(k+1)$-crossing-free $k$-alternation-free sets of edges not contained in $k$-pseudotriangulations (see Fig. 18). Thus, we still miss a simple condition to characterize multipseudotriangulations of points in general position:

Question 51 Characterize primals of multipseudotriangulations.

Another question related to the primal of a multipseudotriangulation would be to determine whether for every point set there exists a multipseudotriangulation that looks as simple as possible. When $k=1$, we know that every point set in general position has:

(i) a pointed pseudotriangulation consisting only of triangles and four-sided pseudotriangles [21];

(ii) a pointed pseudotriangulation whose maximum degree is at most five [21]. 


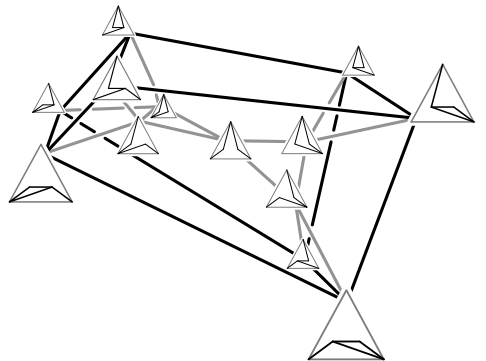

(a)

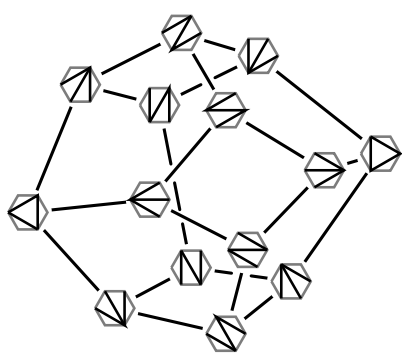

(b)

Fig. 38 (a) The polytope of pointed pseudotriangulations of a set of five points. (b) The 6-dimensional associahedron

Question 52 Does every point set in general position have a $k$-pseudotriangulation with only "simple" stars (resp. only vertices with "little" degree)?

In the previous question, "simple" may be interpreted either as "with exactly $2 k+1$ corners" or as "with at most $2 k+t$ edges" (for a minimal $t$ ). Similarly, "little" means smaller than a constant (as small as possible).

Diameter of the Graph of Flips The graph of flips on $k$-pseudotriangulations of a pseudoline arrangement $L$ is connected, and an easy inductive argument shows that its diameter at is most quadratic in $|L|$. For certain particular cases, even better bounds are known:

(a) The diameter of the graph of flips on pointed pseudotriangulations of a set of $n$ points is at most $O(n \ln n)$ [4].

(b) The graph of flips on the $k$-triangulations of the $n$-gon has diameter bounded by $2 k(n-2 k-1)[25,28]$.

(c) For triangulations of the $n$-gon, the diameter is exactly $2 n-10$ [41].

Question 53 What is the (asymptotic) diameter of the flip graph?

Polytopality Let $\mathcal{S}$ be the support of a pseudoline arrangement. Let $\Delta(\mathcal{S})$ denote the simplicial complex of subsets of contact points of pseudoline arrangements supported by $\mathcal{S}$. Our results ensure that $\Delta(\mathcal{S})$ is an abstract polytope whose ridge graph is the graph of flips (see the discussion in [7, Sect. 2.2]). When $\mathcal{S}$ is the first kernel of the dual pseudoline arrangement of a point set of the Euclidean plane, it turns out that this abstract polytope can be realized effectively as a polytope of $\mathbb{R}^{d}$ (where $d$ is the number of flippable edges), which is the polar of the polytope of pointed pseudotriangulations of [36]. An example of this polytope is presented in Fig. 38(a). When the points are in convex position, this polytope is the associahedron (see Fig. 38(b)). This leads to the following question:

Question 54 Is $\Delta(\mathcal{S})$ the boundary complex of a polytope? 
Fig. 39 A non-stretchable arrangement of nine pseudolines (left) represented on the projective plane to show its symmetries. Remove the center point to obtain the arrangement in the Möbius strip. A pseudotriangulation of it (right)

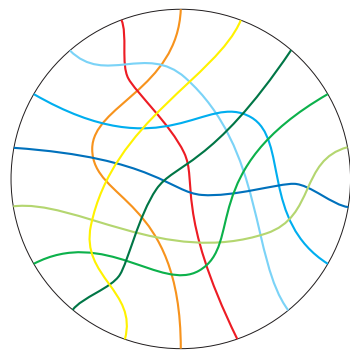

This question specializes for pseudotriangulations and multitriangulations to the following interesting questions:

(a) Is the graph of flips on pseudotriangulations of a point set polytopal? Since [36] answers positively for Euclidean point sets, this question only remains open for non-stretchable arrangements. We have represented in Fig. 39 a pseudotriangulation of the smallest non-stretchable simple pseudoline arrangement (the nonPappus pseudoline arrangement). Since this arrangement is symmetric under the dihedral group $D_{6}$, it could be worked out with methods similar to those in [8].

(b) Is the graph of flips on $k$-triangulations of the $n$-gon polytopal? Jonsson proved that the simplicial complex of $(k+1)$-crossing-free sets of $k$-relevant diagonals of the $n$-gon is a combinatorial sphere [19]. However, except for little cases, the question of the polytopality of this complex remains open. We refer to [8, 28] and [26, Sect. 4.3] for a detailed discussion on this question.

Remark 55 Since the submission of this paper, our knowledge on this question has progressed. In [43], Stump made the connection between the multitriangulations and the type $A$ subword complexes defined in [22]. These simplicial complexes are precisely the simplicial complexes $\Delta(\mathcal{S})$ defined above. It implies in particular that

- these simplicial complexes are either topological spheres or topological balls [22], and

- Question 54 is the special type $A$ case of Question 6.4 in [22].

We close this discussion by mentioning the related construction in [29]. It associates to each sorting network $\mathcal{N}$ its so-called brick polytope whose vertices correspond to certain pseudoline arrangements with contact points supported by $\mathcal{N}$. For certain well-chosen networks, the brick polytope specializes to Hohlweg and Lange's realizations of the associahedron [18]. This construction answers Question 54 positively for a certain class of supports $\mathcal{S}$. It was moreover extended recently to spherical subword complexes of any finite type in [30].

Number of Multipseudotriangulations In his paper [20], Jonsson proved that the number of $k$-triangulations of the $n$-gon is equal to the determinant $\operatorname{det}\left(C_{n-i-j}\right)_{1 \leq i, j \leq k}$ (where $C_{m}=\frac{1}{m+1}\left(\begin{array}{c}2 m \\ m\end{array}\right)$ denotes the $m$ th Catalan number). This determinant also counts non-crossing $k$-tuples of Dyck paths of semi-length $n-2 k$ (see [28] and [26, Sect. 4.1] for a more detailed discussion). It raises the following question: 
Fig. 40 The beam arrangement of a 2-triangulation of the octagon. Each pseudoline is a lattice path
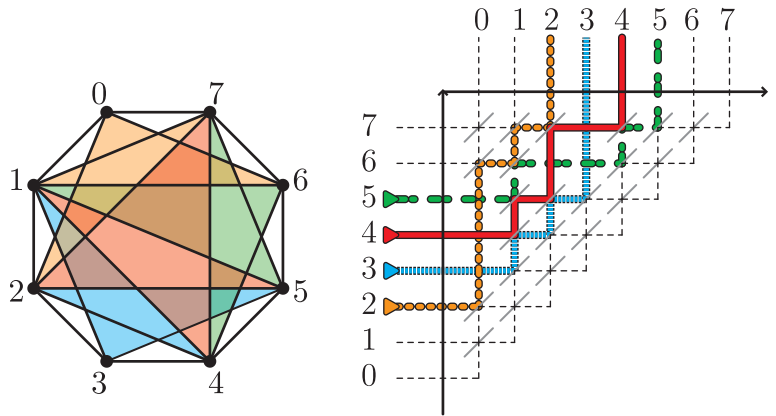

Question 56 Find an explicit bijection between Dyck multipaths and multitriangulations.

Our interpretation of multitriangulations in terms of pseudoline arrangements naturally associates to a $k$-triangulation of the $n$-gon a set of $n-2 k$ lattice paths as follows. Let $T$ be a $k$-triangulation of the $n$-gon. For each edge $(u, v)$ of $T$ (with $u<v$ ), we place a mirror at the grid point of coordinates $(u, v)$. This mirror is a double faced mirror parallel to the diagonal $x=y$ so that it reflects a ray coming from $(-\infty, v)$ to a ray going to $(u,+\infty)$, and a ray coming from $(u,-\infty)$ to a ray going to $(+\infty, v)$. Furthermore, for $1 \leq i \leq n-2 k$, we place a light beam at $(-\infty, k-1+i)$ pointing horizontally. We obtain $n-2 k$ beams which reflect on the mirrors of $T$ (see Fig. 40).

The results of this paper imply the following properties:

(i) All beams are $x$ - and $y$-monotone lattice paths.

(ii) The $i$ th beam comes from the direction $(-\infty, k-1+i)$ and goes to the direction $(k-1+i,+\infty)$.

(iii) Each beam reflects exactly $2 k+1$ times, and thus, has $k$ vertical segments (plus one vertical half-line) and $k$ horizontal segments (plus one horizontal half-line).

(iv) The beams form a pseudoline arrangement: any two of them cross exactly once.

The $i$ th beam $B_{i}$ "corresponds" via duality to the $k$-star $S_{i}$ of $T$ whose $(k+1)$ th vertex is the vertex $k-1+i$ (in other words, the $k$-star bisected by the line passing through the vertex $k-1+i$ and through the midpoint of $[0, n-1])$. Indeed:

(i) the beam $B_{i}$ is (by duality) the set of all bisectors of $S_{i}$;

(ii) the mirrors which reflect $B_{i}$ are the edges of $S_{i}$; and

(iii) the intersection of two beams $B_{i}$ and $B_{j}$ is the common bisector of $S_{i}$ and $S_{j}$.

Observe that instead of $k$ Dyck paths of semi-length $n-2 k$, the beam arrangement of a $k$-triangulation has $n-2 k$ beams which all have $k$ horizontal steps.

Remark 57 In their recent paper [40], Serrano and Stump also observe the correspondence between multitriangulations and beam arrangements (called "reduced pipe dream" in their paper). Starting from this correspondence, they provide an explicit bijection between multitriangulations and Dyck multipaths. We refer to [38, 40, 43] and the references therein. 
Another interesting question concerning the number of multipseudotriangulations would be to determine what point sets give the maximum and minimum number of multipseudotriangulations. For example, when $k=1$, every point set in general position has at least as many pointed pseudotriangulations as the convex polygon with the same number of points [1].

Question 58 What point sets have the maximum and minimum number of multipseudotriangulations?

Decomposition of a $k$-Pseudotriangulation We have seen in Sect. 5 that certain multipseudotriangulations can be decomposed into iterated multipseudotriangulations. Remember, however, that there exist irreducible multipseudotriangulations (Example 35).

Question 59 Characterize (completely) decomposable multipseudotriangulations.

The same question can be asked in a more algorithmical flavor:

Question 60 How can we decide algorithmically whether a $k$-pseudotriangulation is decomposable?

Obviously, a brute-force algorithm is not considered as a good solution. A good way to test efficiency of the answers to Questions 59 and 60 would be to prove/ disprove that Example 35 is the minimal irreducible 2-triangulation (or, in other words, that any 2-triangulation of an $n$-gon, with $n \leq 14$, contains a triangulation).

Remember also that when a multipseudotriangulation is decomposable, the graph of partial flips is not necessarily connected. In particular, finding all decompositions of a multipseudotriangulation cannot be achieved just by searching in the graph of partial flips.

Question 61 How can we enumerate all the decompositions of a multipseudotriangulation?

Computing a $k$-Pseudotriangulation An initial pseudotriangulation of a set of $n$ points can be computed in $O(n \ln n)$ time, using only the predicate of the chirotope. A similar result would be interesting for $k$-pseudotriangulations:

Question 62 Compute an initial $k$-pseudotriangulation of a given (double) pseudoline arrangement, using only its chirotope.

Acknowledgements We thank Francisco Santos for fruitful discussions on the subject (especially for pointing out to us the counter-example of Fig. 22). During his visit at the École Normale Supérieure, Micha Sharir contributed a lot to the improvement of the paper. We thank Jakob Jonsson for pointed out an error in a previous formulation of Lemma 47. Finally, we are grateful to an anonymous reviewer for valuable feedback on the presentation of a preliminary version of this paper.

VP was partially supported by grant MTM2008-04699-C03-02 and MTM2011-22792 of the spanish Ministerio de Ciencia e Innovación, and by European Research Project ExploreMaps (ERC StG 208471). MP was partially supported by the TEOMATRO grant ANR-10-BLAN 0207. 


\section{References}

1. Aichholzer, O., Aurenhammer, F., Krasser, H., Speckmann, B.: Convexity minimizes pseudotriangulations. Comput. Geom. 28(1), 3-10 (2004)

2. Aichholzer, O., Orden, D., Santos, F., Speckmann, B.: On the number of pseudo-triangulations of certain point sets. J. Comb. Theory, Ser. A 115(2), 254-278 (2008)

3. Angelier, P., Pocchiola, M.: A sum of squares theorem for visibility complexes and applications. In: Aronov, B., Basu, S., Pach, J., Sharir, M. (eds.) Discrete and Computational Geometry, the GoodmanPollack Festschrift. Algorithms Combin., vol. 25, pp. 79-139. Springer, Berlin (2003)

4. Bereg, S.: Transforming pseudo-triangulations. Inf. Process. Lett. 90(3), 141-145 (2004)

5. Bereg, S.: Enumerating pseudo-triangulations in the plane. Comput. Geom. 30(3), 207-222 (2005)

6. Björner, A., Las Vergnas, M., Sturmfels, B., White, N., Ziegler, G.M.: Oriented Matroids, 2nd edn. Encyclopedia of Mathematics and Its Applications, vol. 46. Cambridge University Press, Cambridge (1999)

7. Brönnimann, H., Kettner, L., Pocchiola, M., Snoeyink, J.: Counting and enumerating pointed pseudotriangulations with the greedy flip algorithm. SIAM J. Comput. 36(3), 721-739 (2006)

8. Bokowski, J., Pilaud, V.: On symmetric realizations of the simplicial complex of 3-crossing-free sets of diagonals of the octagon. In: Proc. 25th Canadian Conference on Computational Geometry (2009)

9. Capoyleas, V., Pach, J.: A Turán-type theorem on chords of a convex polygon. J. Comb. Theory, Ser. A 56(1), 9-15 (1992)

10. de Bruijn, N.G.: Sorting by means of swappings. Discrete Math. 9, 333-339 (1974)

11. Dress, A.W.M., Klucznik, M., Koolen, J.H., Moulton, V.: $2 k n-\left(\begin{array}{c}2 k+1 \\ 2\end{array}\right):$ note on extremal combinatorics of cyclic split systems. Sém. Lothar. Combin. 47, Article B47b (2001), 17 pp. (electronic)

12. Dress, A.W.M., Koolen, J.H., Moulton, V.: On line arrangements in the hyperbolic plane. Eur. J. Comb. 23(5), 549-557 (2002)

13. Goodman, J.E.: Pseudoline arrangements. In: Handbook of Discrete and Computational Geometry. CRC Press Ser. Discrete Math. Appl., pp. 83-109. CRC, Boca Raton (1997)

14. Grünbaum, B.: Arrangements and Spreads. Conference Board of the Mathematical Sciences Regional Conference Series in Mathematics, vol. 10. American Mathematical Society, Providence (1972)

15. Haas, R., Orden, D., Rote, G., Santos, F., Servatius, B., Servatius, H., Souvaine, D., Streinu, I., Whiteley, W.: Planar minimally rigid graphs and pseudo-triangulations. Comput. Geom. 31(1-2), 31-61 (2005)

16. Habert, L., Pocchiola, M.: Computing pseudotriangulations via branched coverings. Preprint, arXiv:1102.0151. Abbreviated partial version in 20th European Workshop on Comput. Geom. (Seville, 2004), pp. 111-114 (2007)

17. Habert, L., Pocchiola, M.: Arrangements of double pseudolines. Preprint, arXiv:1101.1022. Abbreviated version in 25th Annual ACM Symposium on Comput. Geom. (Aahrus, 2009), pp. 314-323 (2009)

18. Hohlweg, C., Lange, C.E.M.C.: Realizations of the associahedron and cyclohedron. Discrete Comput. Geom. 37(4), 517-543 (2007)

19. Jonsson, J.: Generalized triangulations of the $n$-gon. Unpublished manuscript. An abstract was included in Topological and Geometric Combinatorics, April 2003, Mathematisches Forschungsinstitut Oberwolfach, Report No. 16 (2003)

20. Jonsson, J.: Generalized triangulations and diagonal-free subsets of stack polyominoes. J. Comb. Theory, Ser. A 112(1), 117-142 (2005)

21. Kettner, L., Kirkpatrick, D., Mantler, A., Snoeyink, J., Speckmann, B., Takeuchi, F.: Tight degree bounds for pseudo-triangulations of points. Comput. Geom. 25, 3-12 (2003)

22. Knutson, A., Miller, E.: Subword complexes in Coxeter groups. Adv. Math. 184(1), 161-176 (2004)

23. Knuth, D.E.: The Art of Computer Programming, vol. 3. Addison-Wesley, Reading (1973). Sorting and Searching, Addison-Wesley Series in Computer Science and Information Processing

24. Knuth, D.E.: Axioms and Hulls. Lecture Notes in Computer Science, vol. 606. Springer, Berlin (1992)

25. Nakamigawa, T.: A generalization of diagonal flips in a convex polygon. Theor. Comput. Sci. 235(2), 271-282 (2000)

26. Pilaud, V.: Multitriangulations, pseudotriangulations and some problems of realization of polytopes. Ph.D. thesis, Université Paris 7 \& Universidad de Cantabria (2010)

27. Pilaud, V., Pocchiola, M.: Multipseudotriangulations. In: 25th European Workshop on Comput. Geom., Bruxelles (2009). Extended abstract 
28. Pilaud, V., Santos, F.: Multitriangulations as complexes of star polygons. Discrete Comput. Geom. 41(2), 284-317 (2009)

29. Pilaud, V., Santos, F.: The brick polytope of a sorting network. Eur. J. Comb. 33(4), 632-662 (2012)

30. Pilaud, V., Stump, C.: Brick polytopes of spherical subword complexes: A new approach to generalized associahedra. Preprint, arXiv:1111.3349 (2011)

31. Pocchiola, M.: Horizon trees versus pseudo-triangulations. In: 13th European Workshop on Comput. Geom., Würzburg (1997)

32. Pocchiola, M., Vegter, G.: Order types and visibility types of configurations of disjoint convex plane sets. Technical report, LIENS, Paris (1994)

33. Pocchiola, M., Vegter, G.: Pseudo-triangulations: theory and applications. In: 12th Annual ACM Symposium on Comput. Geom., Philadelphia, pp. 291-300 (1996)

34. Pocchiola, M., Vegter, G.: Topologically sweeping visibility complexes via pseudotriangulations. Discrete Comput. Geom. 16(4), 419-453 (1996)

35. Pocchiola, M., Vegter, G.: The visibility complex. Int. J. Comput. Geom. Appl. 6(3), 279-308 (1996)

36. Rote, G., Santos, F., Streinu, I.: Expansive motions and the polytope of pointed pseudo-triangulations. In: Aronov, B., Basu, S., Pach, J., Sharir, M. (eds.) Discrete and Computational Geometry, The Goodman-Pollack Festschrift. Algorithms Combin., vol. 25, pp. 699-736. Springer, Berlin (2003)

37. Rote, G., Santos, F., Streinu, I.: Pseudo-triangulations-a survey. In: Surveys on Discrete and Computational Geometry. Contemp. Math., vol. 453, pp. 343-410. Am. Math. Soc., Providence (2008)

38. Rubey, M.: Maximal 0-1 fillings of moon polyominoes with restricted chain-lengths and RC-graphs. Preprint, arXiv:1009.3919 (2010)

39. Salzmann, H., Betten, D., Grundhöfer, T., Hähl, H., Löwen, R., Stroppel, M.: Compact Projective Planes. de Gruyter Expositions in Mathematics, vol. 21. de Gruyter, Berlin (1995)

40. Serrano, L., Stump, C.: Maximal fillings of moon polyominoes, simplicial complexes, and Schubert polynomials. Electron. J. Comb. 19 (2012)

41. Sleator, D.D., Tarjan, R.E., Thurston, W.P.: Rotation distance, triangulations, and hyperbolic geometry. J. Am. Math. Soc. 1(3), 647-681 (1988)

42. Streinu, I.: Pseudo-triangulations, rigidity and motion planning. Discrete Comput. Geom. 34(4), 587635 (2005)

43. Stump, C.: A new perspective on $k$-triangulations. J. Comb. Theory, Ser. A 118(6), 1794-1800 (2011)

44. Welzl, E.: Entering and leaving $j$-facets. Discrete Comput. Geom. 25(3), 351-364 (2001) 